\title{
Énergie et environnement en Allemagne
}

Energy and environment in Germany

Energie und Umwelt in Deutschland

\section{Michel Deshaies}

\section{OpenEdition}

\section{Journals}

Édition électronique

URL : http://journals.openedition.org/rge/978

DOI : $10.4000 /$ rge. 978

ISSN : 2108-6478

\section{Éditeur}

Association des géographes de l'Est

Édition imprimée

Date de publication : 1 janvier 2007

ISSN : 0035-3213

\section{Référence électronique}

Michel Deshaies, «Énergie et environnement en Allemagne », Revue Géographique de l'Est [En ligne], vol. 47 / 1 | 2007, mis en ligne le 21 décembre 2009, consulté le 08 septembre 2020. URL : http:// journals.openedition.org/rge/978; DOI : https://doi.org/10.4000/rge.978

\section{Ce document a été généré automatiquement le 8 septembre 2020.}

Tous droits réservés 


\title{
Énergie et environnement en Allemagne
}

\author{
Energy and environment in Germany \\ Energie und Umwelt in Deutschland
}

Michel Deshaies

\section{Introduction}

1 Depuis la réunification du pays en 1990, la politique énergétique allemande a connu de grandes évolutions résultant de l'influence croissante des lois du marché et des préoccupations environnementales. C'est notamment le souci de l'environnement, en apparence particulièrement important en Allemagne qui, au cours de la dernière décennie, a fortement infléchi la politique énergétique, en particulier dans le domaine de la production d'électricité. Le gouvernement de la coalition rouge-verte, au pouvoir de 1997 à 2005, a ainsi engagé le pays dans la sortie du nucléaire et a parallèlement mis en place un très ambitieux programme de développement des énergies renouvelables, avec principalement l'essor des éoliennes. Ces nouvelles orientations vont cependant rapidement trouver leurs limites et risquent surtout d'avoir des conséquences négatives, non seulement sur le taux de dépendance énergétique du pays, mais aussi sur l'environnement; ce qui pour le moment en tout cas, semble encore constituer une vérité qui dérange.

\section{Les orientations récentes de la politique énergétique allemande}

\section{A. Les enjeux traditionnels de la politique énergétique}

2 À l'image de la plupart des pays européens, l'Allemagne dépend fortement des importations pour son approvisionnement énergétique. Cela est dû essentiellement à la 
nécessité dans laquelle elle se trouve d'importer la quasi-totalité des hydrocarbures (pétrole et gaz naturel) qu'elle consomme et qui représentaient en 2006, $59 \%$ de sa consommation d'énergie primaire (figure 1). Le taux de dépendance énergétique du pays était ainsi de $62 \%$ en 2006, assez sensiblement supérieur par exemple, à celui d'un pays comme la France (51\%), pourtant tout aussi dépourvu d'hydrocarbures et qui, depuis 2004, n'extrait plus de charbon1. L'essentiel de l'énergie produite en Allemagne correspond à la production d'électricité à partir du nucléaire et du lignite qui, ensemble, représentent environ un quart de la consommation énergétique du pays. A cela il faut ajouter à peine la moitié de la production des centrales thermiques au charbon, puisque désormais l'Allemagne importe près de $60 \%$ du charbon utilisé pour produire de l'électricité. Le reste, soit $5 \%$ de la consommation énergétique primaire en 2006, est fourni par les énergies renouvelables avec en tête la biomasse, suivie en proportions à peu près équivalentes par l'éolien et l'hydroélectricité.

Figure 1 : Consommation énergétique primaire en Allemagne en $2006: 487$ millions de tonnes équivalent charbon (source : BMWT, 2006).

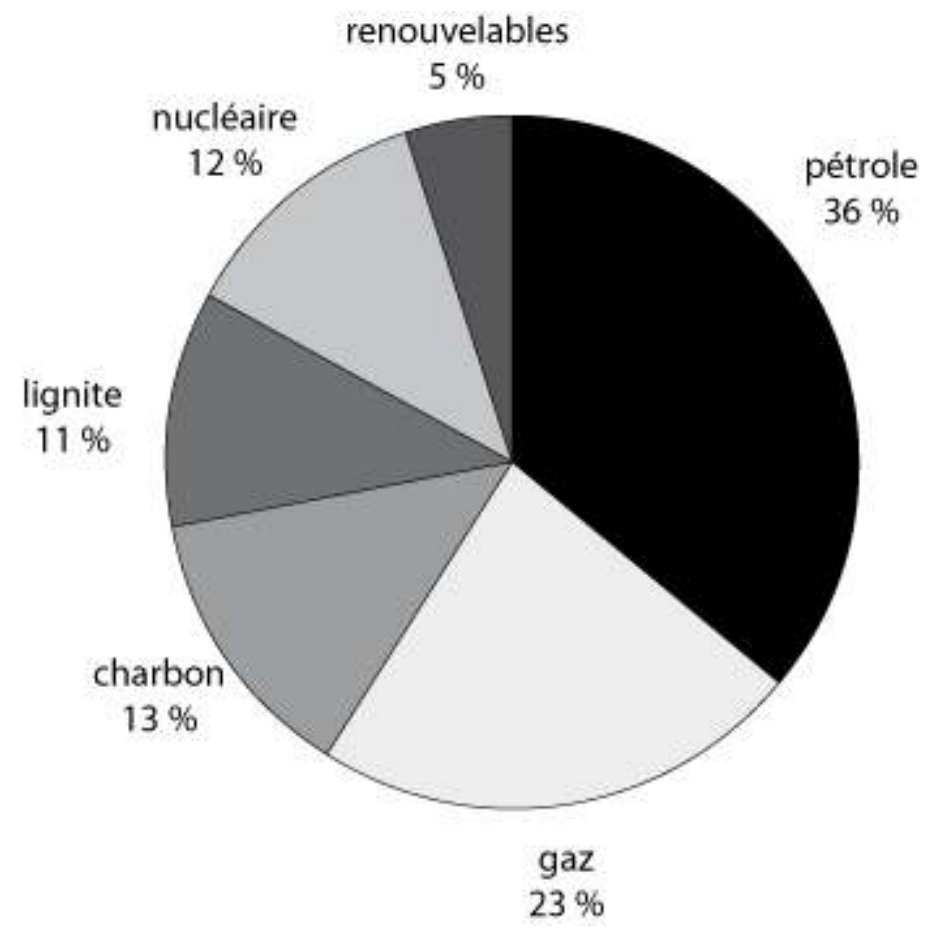

3 La structure actuelle de la production d'électricité qui représente à elle seule environ $40 \%$ de la consommation énergétique primaire s'explique par la prise en compte de trois types de considérations qui se traduisent plus ou moins dans la politique énergétique suivie à l'échelle fédérale ou à l'échelle des Länder :

1. Valoriser au mieux les ressources énergétiques nationales afin de limiter la dépendance visà-vis de l'extérieur.

2. Les impératifs économiques et en particulier les coûts d'exploitation des différentes sources d'énergie.

3. Les considérations environnementales.

4 Si les deux premières constituent en quelque sorte des objectifs classiques de la politique énergétique des États, il en va tout autrement pour la troisième, puisque les préoccupations environnementales n'ont véritablement commencé à influer de 
manière décisive sur la politique énergétique de l'Allemagne, qu'au cours des années 1980.

5 Auparavant, c'était uniquement les deux premiers types de considération qui prévalaient. Dans le cas de l'Allemagne, il faut souligner que pour des raisons historiques, la volonté de limiter la dépendance vis-à-vis de l'extérieur a longtemps joué un rôle dominant. Il faut y voir notamment les conséquences des deux conflits mondiaux dans lesquels l'Allemagne a toujours souffert de sa situation d'État continental, dont les approvisionnements extérieurs pouvaient être facilement coupés par les puissances maritimes. C'est notamment cela qui explique le développement considérable de l'utilisation du lignite, malgré les faibles qualités énergétiques de cette ressource. En effet, durant et après la Première Guerre mondiale, la production de lignite a beaucoup augmenté, afin de permettre dans un premier temps, la satisfaction de la demande croissante d'électricité, puis de compenser la perte des bassins charbonniers de Silésie et de la Sarre. La mobilisation maximale des ressources minières durant la période du IIIe Reich a contribué à développer plus encore l'utilisation du lignite. Enfin, après la Seconde guerre mondiale, la division de l'Allemagne en deux États rivaux, dont l'un, la RDA, était dépourvu de ressources énergétiques, en dehors du lignite, a conduit à une utilisation à outrance de cette ressource qui fournissait en 1989 plus des deux tiers de la consommation énergétique primaire du pays et notamment $85 \%$ de l'électricité.

6 À partir des années 1970, la volonté de limiter la dépendance vis-à-vis de l'extérieur s'appuie aussi sur des considérations économiques, puisque le renchérissement du prix du pétrole conduit les deux États allemands à diminuer leurs importations de produits pétroliers. Cette politique se traduit notamment dans le développement de la production d'électricité, pour laquelle on recourt davantage au charbon et au lignite, en particulier en RDA. À cela vient s'ajouter en RFA la réalisation d'un grand programme de construction de centrales nucléaires qui, dès le milieu des années 1980, fournit près d'un tiers de l'électricité.

7 Cependant, à partir de la fin des années 1980, les considérations économiques vont entraîner une forte réduction de la production de charbon en Allemagne de l'Ouest, tandis que la réunification allemande provoque un effondrement de la production de lignite en Allemagne de l'Est. En effet, la possibilité d'importer du charbon de Pologne, d'Australie ou d'Afrique du Sud à des prix trois fois inférieurs aux coûts d'extraction du charbon allemand finit par condamner une grande partie des charbonnages dont la production est maintenue artificiellement, essentiellement pour des raisons sociales, par un coûteux système de subventions (entre autres, le Kohlepfennig). Aussi, le déclin de la production de charbon, amorcé au milieu des années 1980, s'accélère après la réunification dont le coût rend de plus en plus difficile le soutien à l'industrie charbonnière (ainsi que les fermes recommandations de la commission de Bruxelles); ce qui fait que la production est tombée à environ 25 millions de tonnes actuellement et depuis 2001 les importations sont devenues supérieures à la production.

\section{B. L'importance récente des enjeux environnementaux}

8 En même temps que les impératifs économiques tendaient à prendre le pas sur la question de la dépendance énergétique, les préoccupations environnementales se sont aussi imposées de plus en plus dans le débat sur la politique énergétique. Dès la fin des 
années 1970, les mouvements pacifistes plus ou moins liés aux mouvements écologistes concentrent leurs attaques sur la question hautement symbolique de l'utilisation de l'énergie nucléaire pour la production d'électricité. Les manifestations d'hostilité à la construction de centrales, comme par exemple le surgénérateur de Kalkar en Rhénanie $\mathrm{du}$ Nord-Westphalie, se multiplient. Il est vrai aussi que la querelle n'est pas seulement alimentée par les questions environnementales, mais se joue également sur le plan du fédéralisme. En effet, selon "l'Atomrecht ", les centrales nucléaires sont du ressort du gouvernement fédéral à Berlin ; ce qui fait que la Rhénanie du Nord-Westphalie, le Land $\mathrm{du}$ charbon et du lignite, ne veut pas de centrale nucléaire sur son territoire. Enfin, l'accident survenu le 26 avril 1986 à la centrale de Tchernobyl en Ukraine apporte un argument décisif aux adversaires de l'énergie nucléaire. Cette catastrophe, à l'origine d'un nuage radioactif qui traverse toute l'Europe, provoque un choc dans l'opinion publique et un tournant dans la politique énergétique. L'autorisation de mise en service de la centrale nucléaire de Kalkar est annulée par le Landtag, tandis que la centrale nucléaire de Mülheim-Kärlich près de Coblence (Rhénanie-Palatinat) est arrêtée. Les deux derniers des 19 réacteurs construits en RFA sont mis en service en 1988. Cependant, le principe même de l'utilisation de l'atome pour la production d'électricité n'est pas remis en cause.

L'arrivée au pouvoir en 1997 de la coalition rouge-verte constituée par le parti social démocrate et les écologistes (les "Grünen»), a marqué une nouvelle étape décisive pour la prise en compte des questions environnementales dans la politique énergétique. Le gouvernement allemand a ainsi décidé de sortir du nucléaire en faisant voter une loi entrée en vigueur le 27 avril 2002. Suivant les termes de la « loi pour la cessation ordonnée de l'utilisation de l'énergie nucléaire pour la production industrielle d'électricité ", les 19 réacteurs en service en Allemagne doivent progressivement cesser leur activité entre 2003 et 2020 (HEURIAUX, 2002). Ce tournant dans la politique énergétique allemande est d'ailleurs loin d'être isolé puisque, à la même époque, la Belgique annonce qu'elle ne remplacera pas ses sept réacteurs nucléaires actuellement en service. Enfin, en Espagne, le gouvernement socialiste annonce un moratoire sur le nucléaire, tandis que le Royaume-Uni, où a été mis en service le premier réacteur nucléaire civil du monde en 1956, prévoit également d'arrêter progressivement les réacteurs les plus anciens. Le club des pays européens qui ont refusé (Italie, Autriche) ou qui avaient déjà annoncé leur sortie du nucléaire (Suède) semble ainsi en voie d'élargissement important.

10 Un autre volet essentiel de la prise en compte de l'environnement dans la politique énergétique découle de la signature en 1997 du protocole de Kyoto. L'Allemagne a en effet été l'un des principaux promoteurs de ce protocole par lequel les États industrialisés signataires s'engagent à diminuer globalement leurs émissions de gaz à effet de serre de 5,2\% entre 1990 et 2008/2012; l'objectif étant de diminuer l'accroissement de l'effet de serre afin de limiter l'ampleur du changement climatique constaté depuis plusieurs décennies. Après la signature de la Russie à la fin de l'année 2004, le protocole de Kyoto est entré en vigueur le 16 février 2005. Aux termes de cet accord, c'est en fait l'Union européenne (à 15 à l'époque) qui s'est engagée à réduire ses émissions de $8 \%$. Étant donné la diversité des situations à l'intérieur de l'Union européenne, les pays membres ont négocié un programme sur le changement climatique (European Climate Change Programme) dans le cadre duquel ils se sont vus 
fixer des objectifs très différents tenant compte de l'état de développement de l'industrie et du système de production énergétique.

11 Ainsi, alors que la France doit seulement maintenir ses émissions de gaz à effet de serre au niveau de 1990 et que l'Italie doit les réduire de 6,5\%, c'est précisément l'Allemagne qui supporte l'effort principal puisqu'elle s'est engagée à réduire ses émissions de $21 \%$. Or, comme l'Allemagne est aussi le plus gros émetteur de gaz à effet de serre (environ un quart de l'UE à 15), cela représente les trois quarts de la diminution à laquelle l'Union européenne s'est engagée; c'est dire le rôle décisif que joue la politique énergétique allemande pour permettre à l'Union européenne d'atteindre les objectifs de Kyoto. Or, l'Allemagne est d'ores et déjà pratiquement parvenue à honorer ses engagements; ce qui s'explique en fait essentiellement par la désindustrialisation survenue dans les nouveaux Länder après la réunification allemande. En effet, la forte diminution des émissions de gaz à effet de serre en Allemagne s'explique en majeure partie par la diminution drastique de la production de lignite en ex-RDA, passée en quelques années de 300 à environ 85 millions de tonnes par an et par l'effondrement des industries, notamment les plus polluantes comme la chimie et la fermeture de nombreuses centrales thermiques. C'est d'ailleurs un processus comparable, quoique moins rapide, qu'ont connu les pays d'Europe centrale nouvellement entrés dans l'Union européenne, puisqu'ils dépassent tous largement l'objectif qui leur est assigné de réduire de $8 \%$ leurs émissions de gaz à effet de serre. Leur contribution sera d'ailleurs certainement décisive pour permettre à l'Union européenne d'atteindre l'objectif d'une diminution de $8 \%$ car, en dehors de l'Allemagne, de la France, du Royaume-Uni et de la Grèce, aucun pays de l'ancienne Union européenne à 15 n'est en passe d'honorer ses engagements, bien au contraire. C'est le cas par exemple de l'Italie et de l'Autriche qui devraient diminuer leurs émissions respectivement de 6,5\% et de $13 \%$ et qui les ont en fait augmentées de 11,6 à $16,6 \%$ entre 1990 et 2003, et surtout de l'Espagne dont l'essor économique a provoqué une augmentation de plus de $40 \%$ de ses émissions de gaz, alors que celle-ci devrait se limiter à $15 \%$ (figure 2). Aussi, l'Union européenne à 15 n'est-elle parvenue à diminuer ses émissions que de 1,7\% entre 1990 et 2003. Mais si l'on ajoute les 10 pays entrés dans l'Union en 2004, on atteint une diminution totale de tout juste $8 \%$, soit exactement l'objectif fixé par le protocole de Kyoto. 
Figure 2 : Objectifs et évolutions réelles des émissions de gaz à effet de serre des pays membres de l'Union européenne entre 1990 et 2003 (source : EEA 2005).

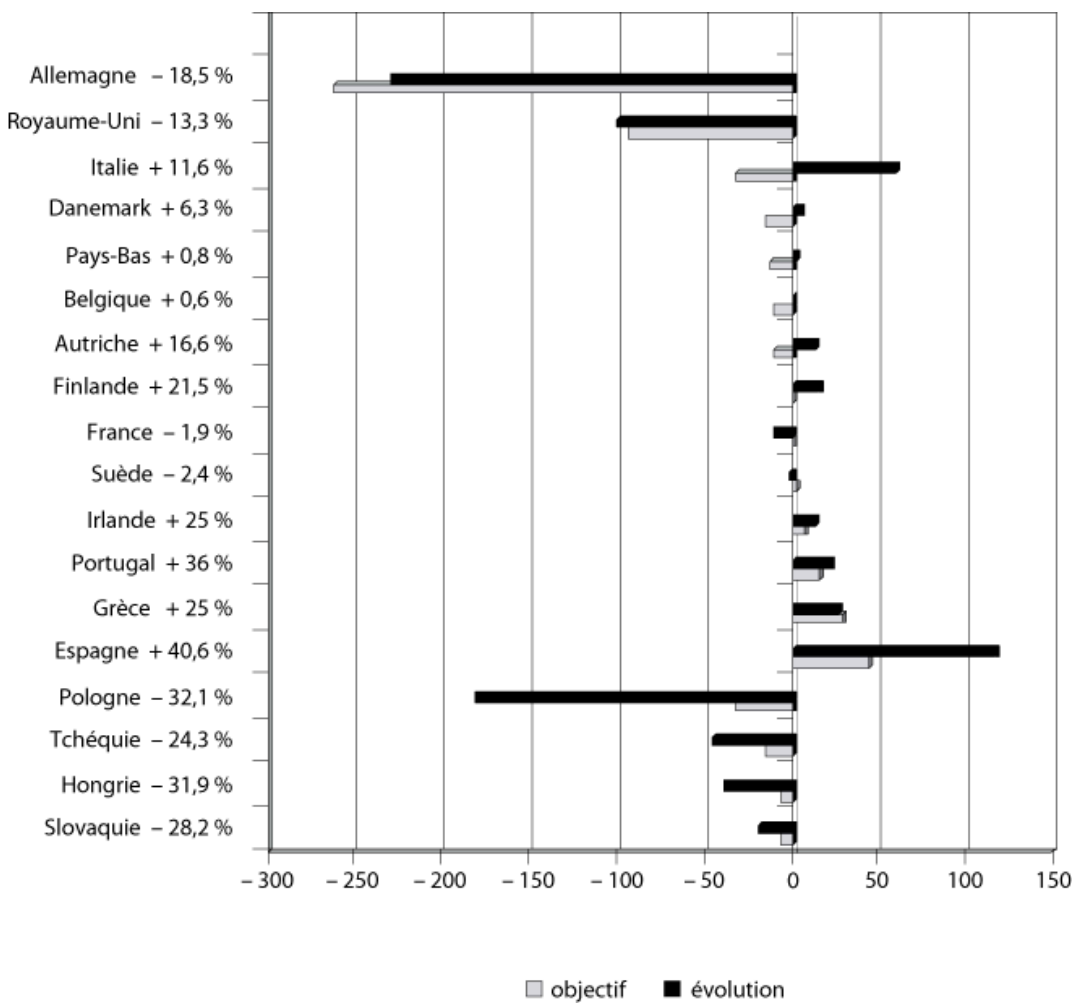

Les chiffres suivant les noms de pays correspondent aux pourcentages d'évolution par rapport à 1990. L'axe des abscisses est gradué en millions de tonnes d'équivalent $\mathrm{CO}_{2}$.

Encore faudrait-il désormais s'y tenir ; ce qui ne paraît pas certain car les marges de manœuvre sont réduites. En effet, étant donné que les pays d'Europe centrale et l'Allemagne orientale ont d'ores et déjà réalisé le gros de leur restructuration industrielle, il ne faut pas s'attendre de ce côté à une diminution des émissions de gaz à effet de serre d'ampleur importante, comme celle déjà réalisée depuis 1990. A l'inverse, les progrès économiques réalisés par les pays d'Europe centrale ont plutôt pour effet de développer la circulation automobile qui a plus que doublé, ainsi que la consommation énergétique des ménages pour le moment encore très inférieure à celle des pays plus riches de l'ancienne Union à 15 (DESHAIES 2006). En fait, l'augmentation constante et rapide des transports automobiles et de la consommation d'électricité rend de plus en plus difficile la réalisation de l'objectif de baisse des émissions de gaz à effet de serre, dont certains comme le dioxyde de carbone ont tendance à nettement augmenter depuis 2000.

13 Aussi, dans les milieux écologistes, on appelle à un changement radical du système économique et des comportements concernant la consommation énergétique, afin de s'inscrire dans l'optique du "développement durable». Il s'agit en l'occurrence d'engager une politique volontariste de réduction de la consommation d'énergie et de développement des énergies renouvelables. Parmi tous les États européens, l'Allemagne est incontestablement celui qui a développé la politique la plus ambitieuse pour l'accroissement de l'efficacité de la consommation énergétique et le développement des énergies renouvelables. 


\section{Des perspectives très ambitieuses de réduction de la consommation énergétique}

Les perspectives d'évolution de la consommation énergétique en Allemagne ont fait l'objet de nombreuses études commanditées par l'ancien gouvernement de la coalition rouge-verte afin de déterminer, entre autres, les conséquences de la sortie programmée du nucléaire. Le point commun de toutes ces études, parmi lesquelles se détache le quatrième rapport sur l'énergie (Energiereport IV, EWI/PROGNOS, 2005) et «l'étude sur le développement optimisé des énergies renouvelables" commanditée par le ministère de l'environnement (BMU, DLR, IFEU, WI, 2004), est de prévoir une réduction sensible de la consommation énergétique à l'horizon 2020. Elles partent de la constatation que l'efficacité énergétique2 en Allemagne a considérablement progressé depuis 1990, si bien que la croissance économique est désormais découplée de l'évolution de la consommation d'énergie. C'est ainsi qu'entre 1990 et 2005 le PIB a augmenté de $25 \%$, alors que la consommation d'énergie a baissé de 4,5\% (figure 3).

Figure 3 : Découplage entre la croissance du PIB et la consommation d'énergie primaire en Allemagne de 1990 à 2005 (BMWT, 2006).

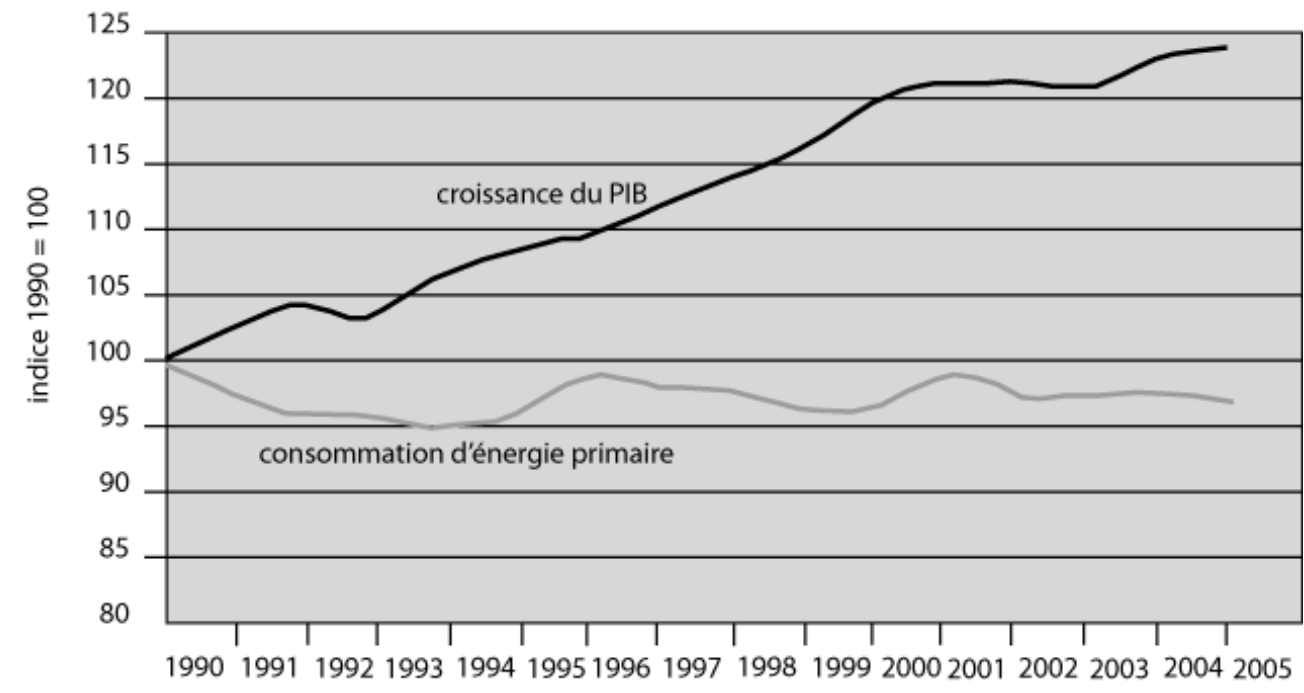

Dans ces conditions, il est envisagé que ces progrès vont s'accentuer dans les années à venir et que l'accroissement de l'efficacité énergétique va permettre de réduire sensiblement la consommation en 2020 : de $9 \%$ suivant le scénario du quatrième rapport sur l'énergie, à $24 \%$ d'après "l'étude sur le développement optimisé des énergies renouvelables ». Parallèlement à la baisse de la consommation, il est prévu que la part des énergies renouvelables va augmenter sensiblement, passant de 4,6 \% en 2005 à $8,3 \%$ en 2020 suivant EWI/PROGNOS (2005) ou même $12 \%$ selon l'étude du BMU (figure 4). Ainsi, grâce à la diminution de la consommation énergétique et à l'essor des énergies renouvelables, il est possible, suivant ces études, de sortir du nucléaire en maintenant la consommation de lignite, de charbon et d'hydrocarbures à un niveau comparable à l'actuel, voire même en la diminuant sensiblement si l'on suit le scénario du ministère de l'environnement. En effet, selon ce scénario "vertueux» la consommation de lignite pourrait être divisée par deux, celle de charbon être réduite de $30 \%$, celle de pétrole de $22 \%$ et celle de gaz naturel de $10 \%$ (figure 4 )! Ces perspectives très ambitieuses sont-elles cependant réalistes? C'est ce qu'il convient 
maintenant d'examiner, en s'attachant dans un premier temps au potentiel de développement des énergies renouvelables.

Figure 4 : Prévisions et scénarios de consommation énergétique en Allemagne en 2020 (en Petajoules). 1 Petajoule équivaut à 23900 tonnes équivalent pétrole (source : EWI/PROGNOS 2005, BMU 2005).

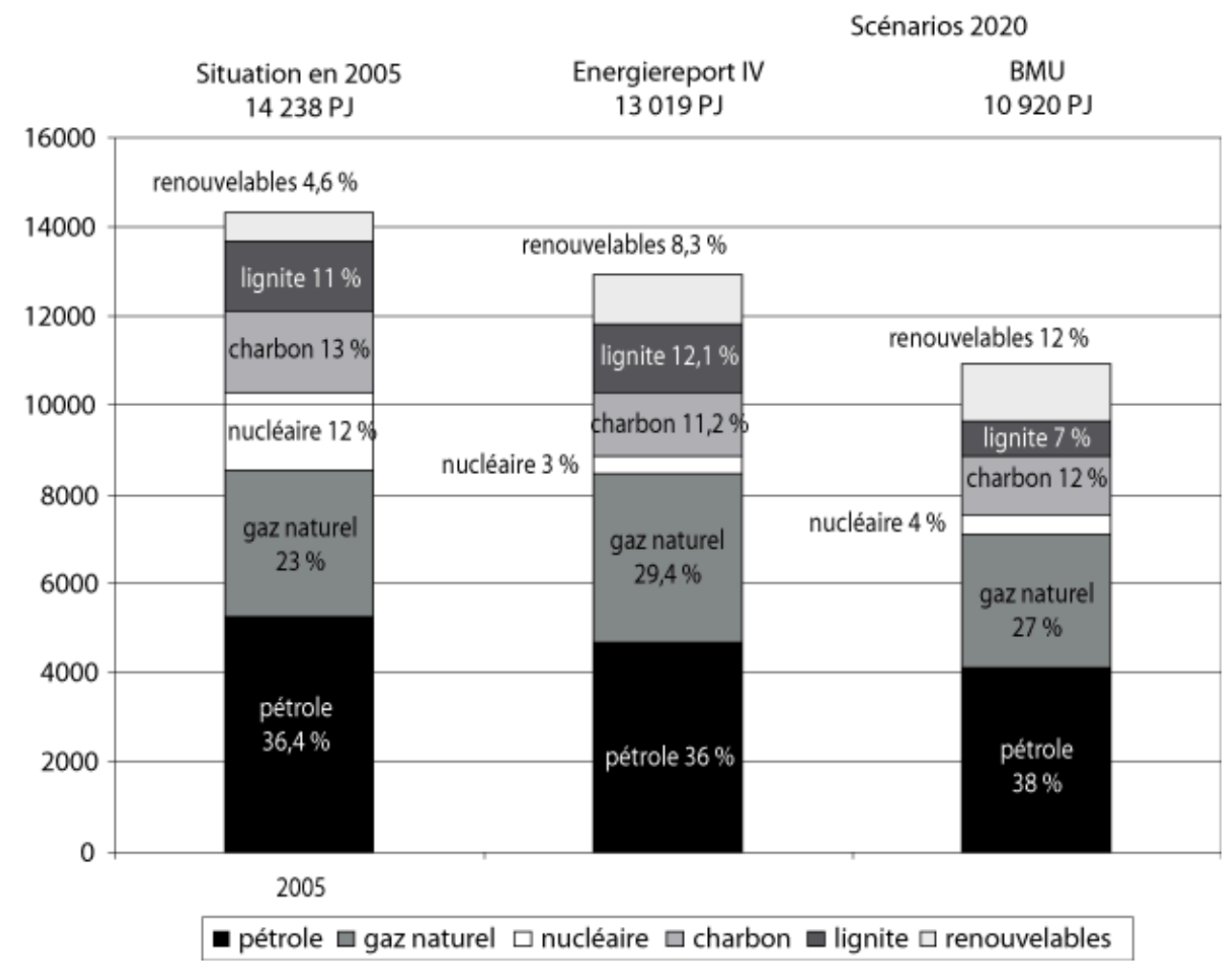

\section{Les limites de l'essor des énergies renouvelables}

\section{A. L'essor spectaculaire des énergies renouvelables}

En quelques années, l'Allemagne est devenue le champion du monde, en chiffres absolus, du développement des énergies renouvelables dont la production a plus que doublé entre 1997 et 2005. Cet essor est particulièrement spectaculaire pour les éoliennes, dont les grandes hélices sont désormais devenues des éléments caractéristiques des paysages allemands (photo 1). Avec 18685 éoliennes et $20622 \mathrm{MW}$ de puissance installée à la fin de l'année 2006, l'Allemagne possède en effet le premier parc éolien du monde dont elle représente environ un tiers. Il faut souligner la rapidité avec laquelle ce parc éolien s'est développé puisqu'en seulement huit ans, de 1998 à 2006, la puissance installée a été multipliée par 7 (figure 5). Avec une production de 29,8 TWh en 2006, soit 4,8\% de l'électricité produite en Allemagne, les éoliennes sont même devenues la première source d'énergie renouvelable puisqu'elles dépassent désormais l'hydroélectricité (figure 6). 
Photo 1 : Un parc éolien sur le plateau de haute Lusace, près de Görlitz (photo M. Deshaies, 2006) : une image devenue classique de l'essor de l'énergie éolienne en Allemagne.

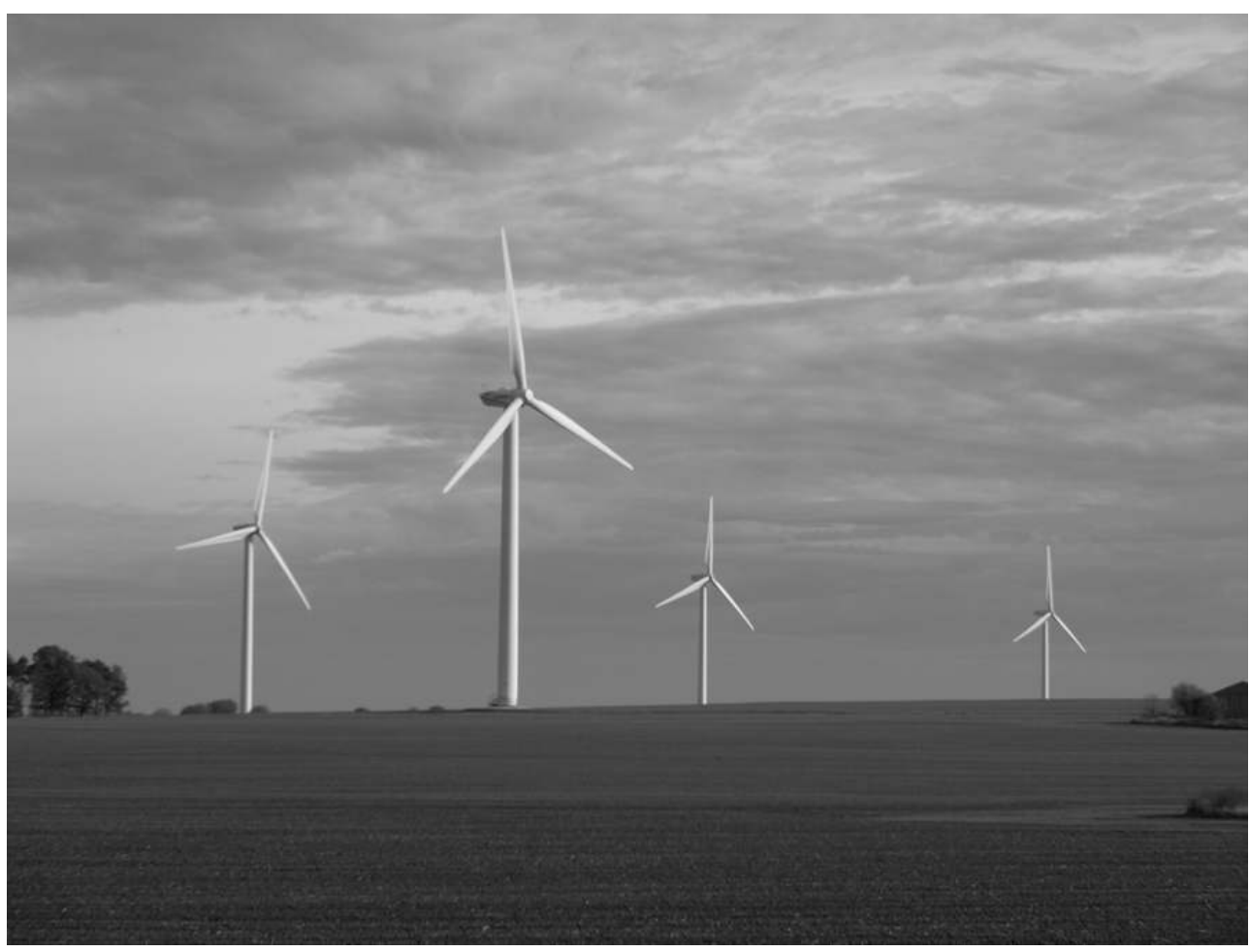

Figure 5 : Croissance des capacités de production d'électricité à partir des éoliennes (puissance installée en MW) (source DEWI).

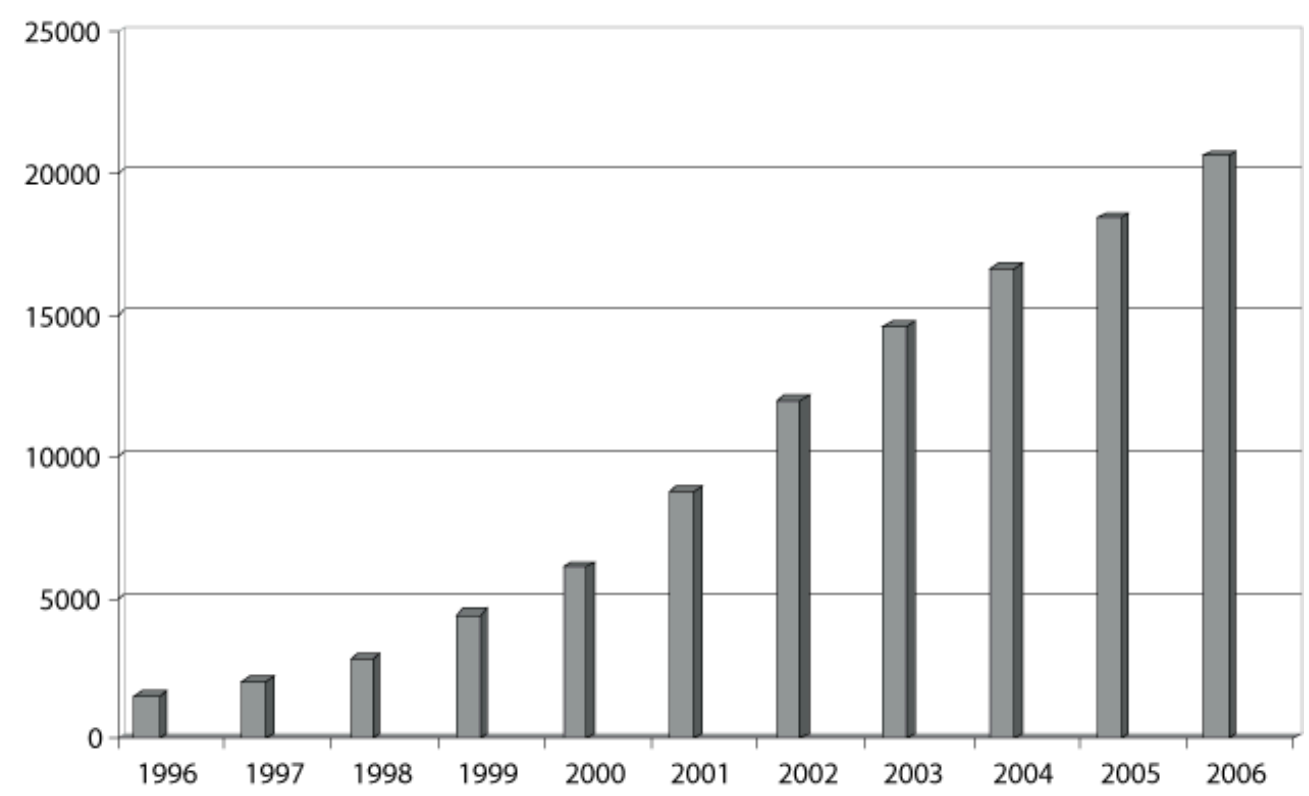


Figure 6 : Évolution de la production d'électricité éolienne et hydraulique en Allemagne (en milliards de kWh) (source DEWI).

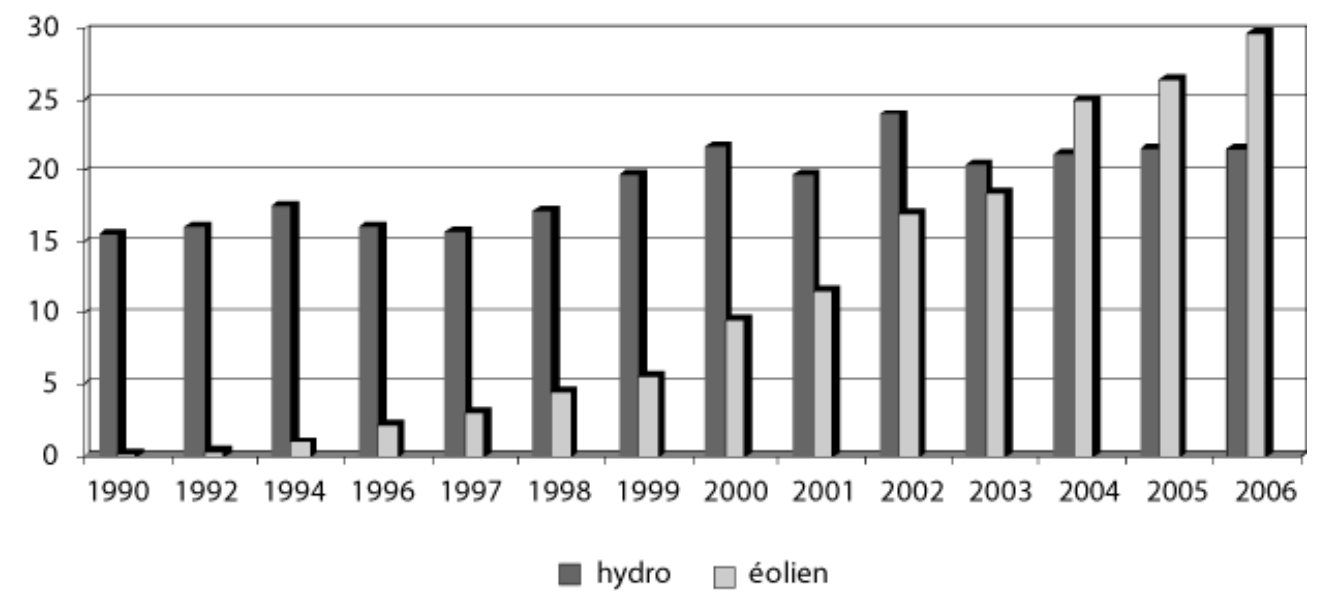

17 Mais ce développement des éoliennes est très inégal suivant les régions et l'on peut ainsi opposer les Länder du Nord (Schleswig-Holstein, Basse-Saxe, Rhénanie du NordWestphalie, Mecklembourg-Poméranie, Brandebourg, Saxe-Anhalt) où se concentrent $83 \%$ de la puissance installée, aux Länder du Sud (Bade-Wurtemberg, Bavière) qui n'ont qu'une part insignifiante des éoliennes (moins de $3 \%$ de la puissance installée). Le tableau 1 montre ainsi que dans le Land le mieux équipé, le Schleswig-Holstein, la densité d'éoliennes est 40 fois plus forte qu'en Bavière (figure 7). Cette différence considérable entre le nord et le sud du pays découle d'une part des contrastes existant dans le potentiel de production éolienne et d'autre part des orientations différentes des politiques énergétiques des Länder. En effet, en raison de la fréquence et de la vitesse plus élevées du vent sur les sites côtiers, ceux-ci offrent un potentiel de production plus élevé que les sites continentaux éloignés des côtes comme en Allemagne du Sud. Une étude de l'ISET3 (2004) a ainsi montré qu'en moyenne entre 1992 et 2003, les éoliennes du Bade-Wurtemberg avaient tourné à plein rendement environ 1000 heures par an contre 1900 heures en Basse-Saxe et même presque 200 heures au Schleswig Holstein. 
Figure 7 : L'énergie éolienne en Allemagne : puissance installée par Land en 2006 et vitesse moyenne du vent (sources: DEWI, BMWT) : 1 : vitesses moyennes du vent, 2 : puissance installée et part du parc éolien dans la puissance installée totale des centrales de production d'électricité du Land, 3 : parc éolien off-shore autorisé, 4 : ligne des 12 miles, 5 : limite de la zone économique exclusive.

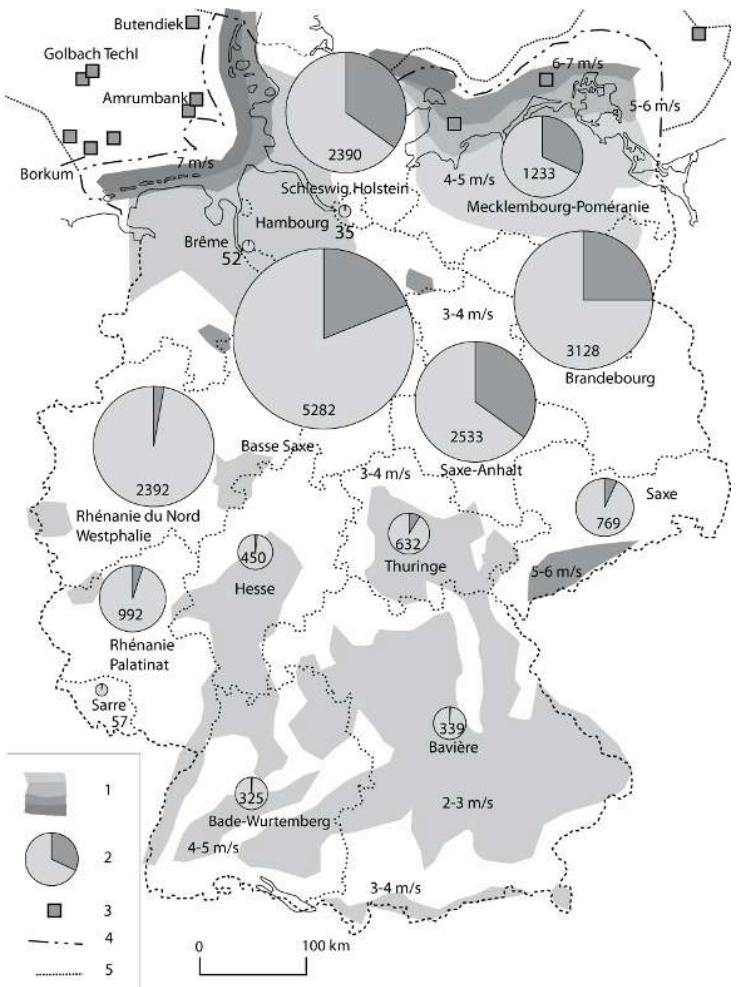

Plus discret que le développement de l'éolien, l'essor de l'utilisation de la biomasse n'en est pas moins spectaculaire et elle constitue de loin la première source d'énergie renouvelable avec plus de la moitié du total. Il s'agit principalement de produire de la chaleur pour les chauffages urbains en brûlant des déchets agricoles, forestiers, municipaux et industriels. Cette énergie représente à elle seule $46 \%$ du total des énergies renouvelables en 2006. Une forme d'utilisation de la biomasse qui a connu un essor récent particulièrement important est la production d'électricité obtenue en brûlant du biogaz, ou des déchets organiques qui servent à générer de la vapeur comme dans les centrales thermiques. Restée insignifiante jusqu'à la fin des années 1990, celleci a depuis lors décuplé pour atteindre $13 \mathrm{TWh}$ en 2006. Elle est en tout cas très supérieure à la production d'électricité à partir de l'énergie solaire, captée par des cellules photovoltaïques. Celle-ci a certes connu un essor impressionnant, puisqu'elle a été multipliée par 20 entre 2001 et 2006 et les toits recouverts de cellules photovoltaïques sont devenus en quelques années un élément assez commun dans les villes et les villages d'Allemagne. Néanmoins, l'électricité produite à partir de l'énergie solaire représentait tout juste 2 TWh en 2006, soit 0,3\% de la consommation. Il est vrai qu'en raison du coût élevé des installations, celle-ci ne représente encore qu'une fraction marginale de l'utilisation de l'énergie solaire, principalement utilisée pour le chauffage des maisons et des chauffe-eau. Ce sont précisément les coûts de fabrication et d'installation des cellules photovoltaïques qui constituent les limites actuelles au développement de cette énergie fortement tributaire des subventions. Au total, les énergies renouvelables ont représenté en 2006, 4,8 \% de la consommation énergétique primaire en Allemagne. 
Figure 8 : La production d'électricité à partir de sources d'énergie renouvelable en Allemagne en $2006: 71,5$ TWh (source : BMU)

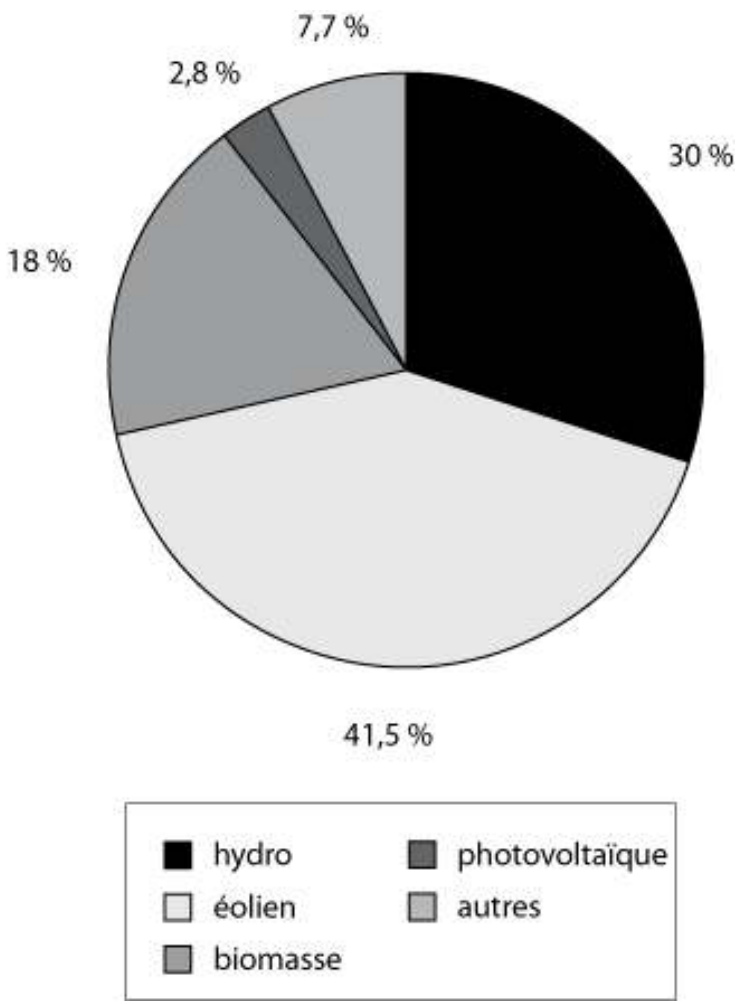

Cet essor remarquable des énergies renouvelables est la conséquence d'une politique volontariste amorcée au début des années 1990 et considérablement renforcée par le gouvernement de la coalition rouge-verte. Celui-ci a mis en place un certain nombre de dispositifs incitatifs destinés à subventionner le recours aux énergies renouvelables qui, pour la plupart, ne sont pas concurrentielles. Ces mesures incitatives sont pour l'essentiel réunies dans la loi sur les énergies renouvelables (Erneuerbare-Energien Gesetz ou EEG) adoptée le 1er avril 2000. Cette loi oblige notamment les électriciens exploitants à racheter prioritairement l'électricité produite à partir des sources d'énergie renouvelables et surtout elle assure une forte augmentation de la rémunération $4 \mathrm{du}$ courant "écologique» (HEURIAUX, 2002). Cela revient à faire subventionner les énergies renouvelables par les autres énergies et en définitive par le consommateur.

L'Allemagne veut ainsi mener une politique exemplaire, à l'avant-garde du plan d'action décidé à l'échelle communautaire et mis en œuvre dans le cadre de la Directive européenne de septembre 2001 qui préconise de faire passer la part des énergies renouvelables dans la production d'électricité, de $14 \%$ en 1997 à $22 \%$ en 2010 ; ce qui revient à doubler la part des énergies renouvelables dans la consommation énergétique primaire. L'objectif que s'est assignée l'Allemagne est à long terme particulièrement ambitieux, puisqu'il s'agit de faire passer la part des énergies renouvelables dans la consommation totale d'énergie de $5 \%$ en 2004 à $50 \%$ à l'horizon 2050. Pour atteindre cet objectif, on compte beaucoup sur le développement de l'utilisation des énergies renouvelables pour la production d'électricité. Leur part qui atteint 11,6\% en 2006 a ainsi doublé depuis 1999 et devrait être d'au moins $20 \%$ en 2020. Dans cette optique, on prévoit de continuer à développer très fortement l'énergie éolienne dont la part devrait 
être portée à $10 \%$ de la production d'électricité en 2020 et à $25 \%$ en 2025 . Lors du récent sommet de Bruxelles des 8 et 9 mars 2007, l'Allemagne a même réussi à faire adopter par l'Union européenne l'objectif très ambitieux de $20 \%$ du bilan énergétique en énergies renouvelables.

\section{B. Les perspectives limitées de croissance de l'énergie éolienne}

21 Cet essor spectaculaire de l'énergie éolienne doit cependant être tempéré car l'une des principales limites à son développement est son extrême dépendance des conditions météorologiques et donc la variabilité imprévisible de la production qui complique l'équilibre du réseau. Or, il se trouve qu'avec l'essor rapide de la capacité installée à partir de 1997, l'essentiel des éoliennes a été construit sur des sites relativement peu productifs. En effet, alors que jusqu'en 1995 les sites côtiers occupaient une place prédominante, depuis lors on a implanté presque les deux tiers des nouvelles éoliennes à l'intérieur des terres, dans la plaine du nord, alors que la capacité de production y est de deux à trois fois inférieure à celle des éoliennes situées sur le littoral. Comme $96 \%$ de la puissance installée actuelle l'a été après 1995, ce sont donc presque les deux tiers des éoliennes qui se trouvent dans les sites relativement peu productifs de la plaine du nord.

Aussi, l'énergie éolienne se signale-t-elle par un écart très important entre la puissance installée (20622 MW fin 2006, soit $10 \%$ de la puissance totale des centrales) et la production effective de courant électrique ( $4,8 \%$ du total). En d'autres termes, la capacité totale des éoliennes allemandes équivaut à celle de 22 réacteurs nucléaires de $950 \mathrm{MW}$, mais leur production atteint tout juste celle de 3 réacteurs). Ceci est dû au fait qu'en moyenne les éoliennes ne fonctionnent au mieux que pendant un peu plus de 2000 heures par an, tandis que les centrales au lignite tournent pendant plus de 7000 heures et les centrales nucléaires plus de 7200 heures Si l'on ajoute à cela que les bons sites pouvant être équipés se font rares et qu'il s'agit d'une énergie jusque là non compétitive, dont l'essor n'est toujours possible que par de généreuses subventions, on peut raisonnablement se demander si l'objectif affiché par l'ancien ministre de l'environnement, Jürgen Trittin, de porter la part de l'électricité éolienne à $10 \%$ en 2020 , puis à $25 \%$ en 2025 n'était pas excessivement ambitieux.

De plus, la poursuite du développement de l'énergie éolienne se heurte à deux obstacles: la rareté des sites intéressants et les critiques de plus en plus vigoureuses des riverains qui reprochent aux éoliennes d'être bruyantes et inesthétiques. En effet, l'implantation brutale des champs d'éoliennes, notamment dans le nord de l'Allemagne, a provoqué la constitution de plusieurs centaines d'associations locales de citoyens inquiets de l'impact environnemental négatif de ces pylônes qui, d'après eux, défigurent les paysages. En raison des résistances de plus en plus grandes que rencontrent les projets de nouvelles implantations, on cherche désormais à suivre l'exemple du Danemark, en développant la production off-shore qui a aussi l'avantage de bénéficier d'une capacité de production bien supérieure. En effet, au lieu de 2000 heures par an pour les meilleures localisations sur le continent, les sites off-shore permettent d'atteindre près de 3500 heures Mais la réalisation de telles installations en pleine mer, où elles devront résister à des conditions météorologiques extrêmes, ne risque-t-elle pas d'augmenter les coûts de construction dans des proportions tellement importantes que le courant produit sera d'un coût prohibitif? Il faut souligner que, 
jusqu'à présent, la réalisation des premiers parcs éoliens off-shore autorisés a été différée en raison de problèmes de financement ou d'autorisation concernant le tracé des lignes à haute tension qui doivent acheminer le courant produit sur le continent et traverser les parcs nationaux de la mer du Nord. C'est ainsi qu'après trois années de négociations, un premier parc expérimental d'une douzaine d'éoliennes devrait être réalisé en 2008, à $45 \mathrm{~km}$ au large des côtes de l'île de Borkum (http://www.offshorestiftung.de). Si les essais sont concluants, le parc éolien offshore devrait comporter 208 éoliennes pour une puissance installée totale de $1000 \mathrm{MW}$, soit juste la capacité d'un réacteur nucléaire. La construction d'un autre parc éolien offshore (le Butendiek) a été autorisée en décembre 2002, mais les travaux qui, initialement, devaient être réalisés en 2006, n'ont toujours pas commencé. Le parc éolien qui doit comporter 80 éoliennes de $3 \mathrm{MW}$ chacune et être implanté à $34 \mathrm{~km}$ à l'ouest de l'île de Sylt, a d'abord été critiqué par l'association écologiste BUND (par ailleurs pourtant partisane de l'énergie éolienne), car il est situé à la limite d’une zone naturelle protégée («Sturmlauf gegen den Ökostrom » (Assaut contre le courant écologique), Spiegel online du 26 août 2003). Mais surtout, ce parc dans lequel 8400 citoyens ont déjà investi, n'a pas encore trouvé, jusqu'à récemment, de partenaire capable de réaliser le projet. Ce n'est qu'en décembre 2006 qu'un accord de partenariat a pu être signé avec une société irlandaise spécialisée dans les parcs éoliens, Airtricity (http://www.butendiek.de). Si cet accord est validé par l'assemblée des actionnaires du Butendiek, le parc off-shore pourrait être terminé en 2009. Malgré les difficultés que rencontrent la réalisation des premiers parcs, il est toujours question d'après la Dena5, d'intensifier le développement des parcs off-shore dont la capacité devrait être portée à $20000 \mathrm{MW}$ (soit 10000 éoliennes de $2 \mathrm{MW}$ ) à l'horizon 2020. A cela, il faut ajouter, toujours selon la Dena, que la poursuite du développement des implantations sur le continent devrait accroître la capacité installée de $28000 \mathrm{MW}$ (au lieu de 20622 en 2006).

Mais en raison du coût financier d'une énergie largement subventionnée, beaucoup jugent aujourd'hui que l'importance de la capacité de production électrique éolienne dont pourrait disposer l'Allemagne en 2020 a été largement surestimée par la Dena. C'est ce que montre par exemple l'étude publiée en septembre 2005 par la Deutsche Physikalische Gesellschaft (DPG), intitulée Klimaschutz und Energieversorgung in Deutschland6 (1990-2020). Selon cette étude, de nombreux problèmes sous-estimés par la Dena, comme celui du financement des nouveaux parcs éoliens, mais aussi la nécessité de créer plusieurs centaines de kilomètres de nouvelles lignes à haute tension pour raccorder au réseau les parcs off-shore, ne permettront pas un développement aussi rapide que prévu. Aussi, la DPG estime que les nouvelles capacités de production éolienne qui pourront être créées d'ici à 2020 ne dépasseront pas un total de $36000 \mathrm{MW}$ (soit la capacité prévue par La Dena en 2015) se partageant entre 9800 MW en off-shore et $26200 \mathrm{MW}$ sur le continent. Dans ces conditions, la production d'électricité éolienne pourrait tripler et atteindre environ 76 TWh en 2020 , soit $14 \%$ de la consommation actuelle. Si l'on ajoute à cela la production d'hydroélectricité que l'on ne peut plus accroître, ainsi que celle d'autres sources d'énergie comme la biomasse et le solaire, on peut envisager selon la DPG, que les énergies renouvelables fournissent entre 98 et 108 TWh à l'horizon 2020, soit 16 à $18 \%$ de la consommation d'électricité de 2005. Mais de toute façon, le potentiel de développement de toutes ces énergies renouvelables est limité, non seulement par les contraintes financières, mais aussi par le fait qu'elles consomment beaucoup d'espace et qu'elles se prêtent mal à l'approvisionnement de régions densément peuplées, aux activités industrielles ou tertiaires consommant 
beaucoup d'électricité et dont la demande fluctue fortement au cours de la journée (Brücher, 2006).

\section{Les conséquences paradoxales de la sortie annoncée du nucléaire}

\section{A. L'incapacité des énergies renouvelables à remplacer la production électronucléaire}

Bien que le gouvernement allemand ait décidé en 2002 la sortie du nucléaire, celui-ci reste encore pour l'heure, la première source de production d'électricité. En effet, depuis 2002 seuls les deux plus petits réacteurs (Stade et Obrigheim) ont été arrêtés, si bien qu'en 2006 les 17 réacteurs en service ont encore produit $26,5 \%$ de l'électricité du pays, juste devant le lignite qui en a fourni $24 \%$ (figure 9). Le prochain réacteur qui sera arrêté en 2007 sera celui de Biblis A (1 $167 \mathrm{MW})$. Puis trois autres réacteurs seront arrêtés en 2009 et d'ici à 2015 c'est une capacité de production de 8351 MW (soit $41 \%$ du potentiel électronucléaire allemand) qui doit être retirée du circuit, les dernières centrales étant mises hors service entre 2020 et 2025. Autrement dit, si l'on traduit cela en termes de production, en 2015, l'électronucléaire devrait fournir 70 milliards de kWh en moins qu'il faudra bien remplacer par une autre source de production.

Figure 9 : Sources de production d'électricité en Allemagne en 2006 (633 TWh).

(source : BMWT)

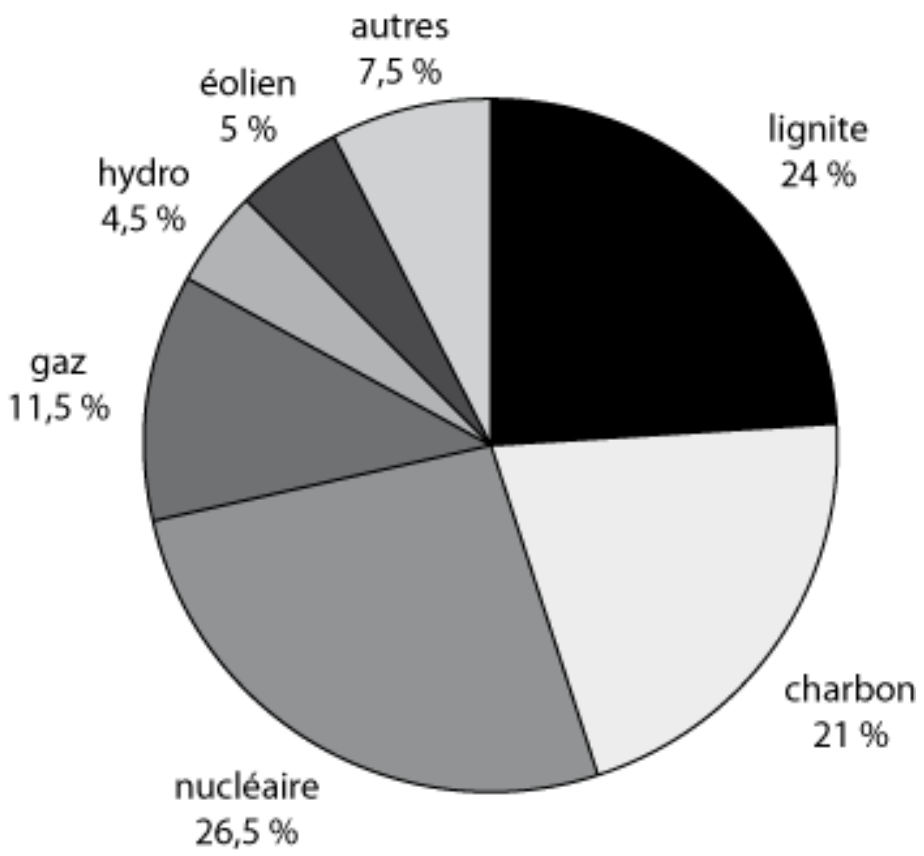

On a vu précédemment qu'il était difficile d'envisager une augmentation de la production d'électricité éolienne supérieure à 50 TWh avant 2020. La différence pourrait certes en partie venir de la biomasse qui, pour l'heure, fournit environ 13 TWh, mais dont la production pourrait doubler ou tripler d'ici à 2015. Par contre, il paraît évident que l'arrêt complet de la plupart des réacteurs nucléaires entre 2015 et 2020 , soit une capacité de production actuelle d'environ $100 \mathrm{TWh}$, ne pourra 
absolument pas être compensé par le développement des énergies renouvelables. A cela, il faut ajouter que la plupart des scénarios envisageant le remplacement de l'énergie nucléaire tablent sur une constance ou du moins une très faible progression de la consommation d'électricité. Or, cela est totalement démenti par l'évolution observée depuis le milieu des années quatre-vingt-dix. En effet, si la consommation d'électricité de l'Allemagne a baissé entre 1990 et 1993 en conséquence de la désindustrialisation dans les nouveaux Länder, depuis lors elle n'a pas cessé d'augmenter, passant de 527 TWh en 1993 à 633 TWh en 2006, soit en moyenne une augmentation de 9 TWh par an (figure 10 et tableau 2). Depuis 2000, l'augmentation de la consommation s'est même accélérée dépassant 57 TWh, soit pratiquement le double de toute la production d'électricité éolienne en 2006. Autrement dit, l'augmentation de la consommation a été en volume plus de trois fois supérieure à celle de l'énergie éolienne (environ 3 TWh par an) qui, à aucun moment, n'a pu couvrir l'accroissement de la demande. Même si le développement de la consommation se poursuit à un rythme beaucoup plus faible au cours des dix prochaines années, cela signifie que le développement du parc d'éoliennes ne pourrait même pas compenser l'augmentation de la consommation.

Figure 10 : Augmentation de la consommation d'électricité et évolution de la production éolienne depuis 1993, année du minimum de consommation depuis 1990 (en TWh).(source : BMWT)

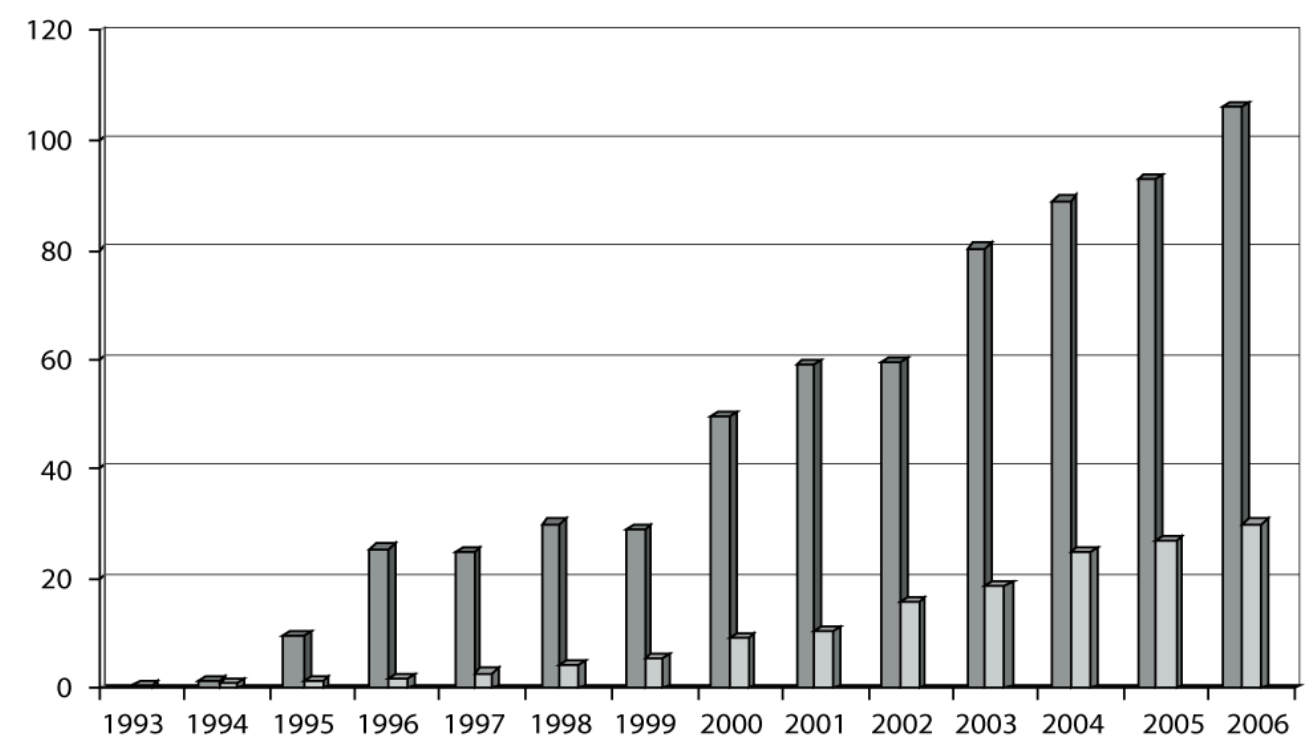

$\square$ cons. électricité $\quad \square$ éolienne

\section{B. L'insurmontable contradiction entre la sortie du nucléaire et les objectifs du protocole de Kyoto}

27 L'incapacité actuelle des sources d'énergie renouvelables à compenser, même partiellement, une forte réduction, et à plus forte raison la suppression de l'énergie nucléaire, conduit à s'interroger sur les conséquences environnementales d'une "sortie» du nucléaire. En effet, en Allemagne, toutes les hypothèses sur le remplacement de l'électricité produite par les centrales nucléaires conduisent à recourir de manière accrue, non seulement aux énergies renouvelables, mais aussi aux 
énergies fossiles, notamment le gaz naturel, le charbon et le lignite. Les scénarios établis récemment (Energiereport IV, EWI/PROGNOS 2005 ; EWI/PROGNOS 2006) par l'Institut d'économie de l'énergie (EWI) de Cologne et par l'Institut de prévision Prognos de Bâle montrent ainsi que, même si la consommation d'électricité diminue sensiblement à l'horizon 2030, la production d'électricité devra toujours être assurée à $71 \%$ par le gaz naturel, le charbon et le lignite, soit un peu plus qu'actuellement (68\%). Les deux scénarios présentés sur la figure 11 divergent seulement sur la place future du charbon. En effet, dans le premier scénario correspondant à l'Energiereport IV, il était envisagé un triplement de la part du gaz naturel par rapport à 2005 ; ce qui se faisait principalement au détriment du charbon réduit alors à $9 \%$ de la production d'électricité. La forte augmentation récente des prix des hydrocarbures a conduit les instituts de prévision à envisager un nouveau scénario (EWI/PROGNOS, 2006) prenant en compte un prix élevé du gaz naturel. Dans cette hypothèse fort probable étant donné le déclin des réserves, la part du gaz naturel resterait sensiblement la même qu'en 2005; si bien que la sortie du nucléaire se traduirait par une sensible augmentation de la part du charbon qui atteindrait alors $31 \%$, au lieu de $22 \%$ en 2005, tandis que le lignite devrait également progresser à $29 \%$. Or, malgré la baisse sensible de la production totale, cela implique que la production d'électricité à partir du lignite et du charbon passe de 290 milliards de kWh actuellement à 350 en 2030.

L'un ou l'autre scénario posent de toute façon des problèmes sérieux pour l'avenir de l'approvisionnement énergétique de l'Allemagne. Dans le premier scénario, le recours accru au gaz naturel, essentiellement importé de Russie, augmente dangereusement la dépendance énergétique du pays, pourtant déjà assez importante ; ce qui n'est pas sans inconvénient comme l'ont montré les épisodes des conflits du gaz entre la Russie et l'Ukraine durant l'hiver 2005-20 067, puis entre la Russie et la Biélorussie en 2006-2007. C'est ainsi qu'en 2004 l'Allemagne a importé de Russie environ 38 milliards de m3 de gaz, soit $42 \%$ de ses importations. Si l'on ajoute à cela qu'avec une part de $34 \%$, la Russie est également de loin le premier fournisseur de pétrole de l'Allemagne, elle apparaît donc d'ores et déjà en situation de forte dépendance énergétique vis-à-vis de Moscou (R. GÖTZ, 2006). Or, dans l'avenir, cette dépendance devrait s'accroître sensiblement, d'une part en raison de l'augmentation de la consommation allemande de gaz, d'autre part en conséquence du recul rapide des réserves de gaz naturel et de pétrole de la Mer du Nord. Aussi, pour acheminer les 60 milliards de m3 par an que l'Allemagne pourrait importer de Russie à l'horizon 2020, il a été décidé conjointement par Moscou et Berlin de construire un nouveau gazoduc direct à travers la Mer Baltique, le NEGP (Nordeuropäische Gaspipeline).

Dans le second scénario, le renforcement de l'emploi du charbon et du lignite pour la production d'électricité est en contradiction avec la politique affichée de développer des sources d'énergies qui soient "umweltfreundlich", c'est-à-dire favorables à l'environnement. En effet, l'utilisation encore massive de ces combustibles fossiles contribue largement au fait que pour sa production d'électricité, l'Allemagne produit aujourd'hui sept fois plus de gaz à effet de serre qu'un pays comme la France qui utilise essentiellement l'énergie nucléaire et l'hydroélectricité. Ainsi, alors que la production d'électricité de l'Allemagne représente environ $20 \%$ de celle de l'ancienne Union européenne à 15 , elle est responsable de près d'un tiers du dioxyde de carbone émis par la production électrique (photo 2). Seuls les pays consommant encore plus de charbon ou de lignite par habitant, comme le Danemark, la Grèce, la Pologne et la République 
tchèque, polluent plus que l'Allemagne. Il est vrai aussi que les centrales thermiques allemandes sont incontestablement les plus performantes d'Europe et l'on compte beaucoup, dans les années à venir, sur un sensible accroissement de leur rendement ; ce qui permettrait d'augmenter la production d'électricité sans consommer davantage de charbon ou de lignite et donc sans rejeter plus de $\mathrm{CO} 2$. C'est ainsi que la nouvelle génération de centrales au lignite mises en service depuis 1998 dans les nouveaux Länder (Lippendorf, Schwarze Pumpe, Buna et Boxberg) et en cours de construction dans la région de Cologne (Niederaussem) atteint un rendement de $43 \%$ contre $35 \%$ pour les centrales construites dans les années soixante-dix.

Photo 2 : La centrale thermique de Boxberg, en Basse Lusace, a été complètement rénovée depuis la réunification allemande (photo M. Deshaies, 2006). Au premier plan, le « Findlingspark » (parc des blocs erratiques) est une initiative originale pour réhabiliter une ancienne exploitation de lignite.

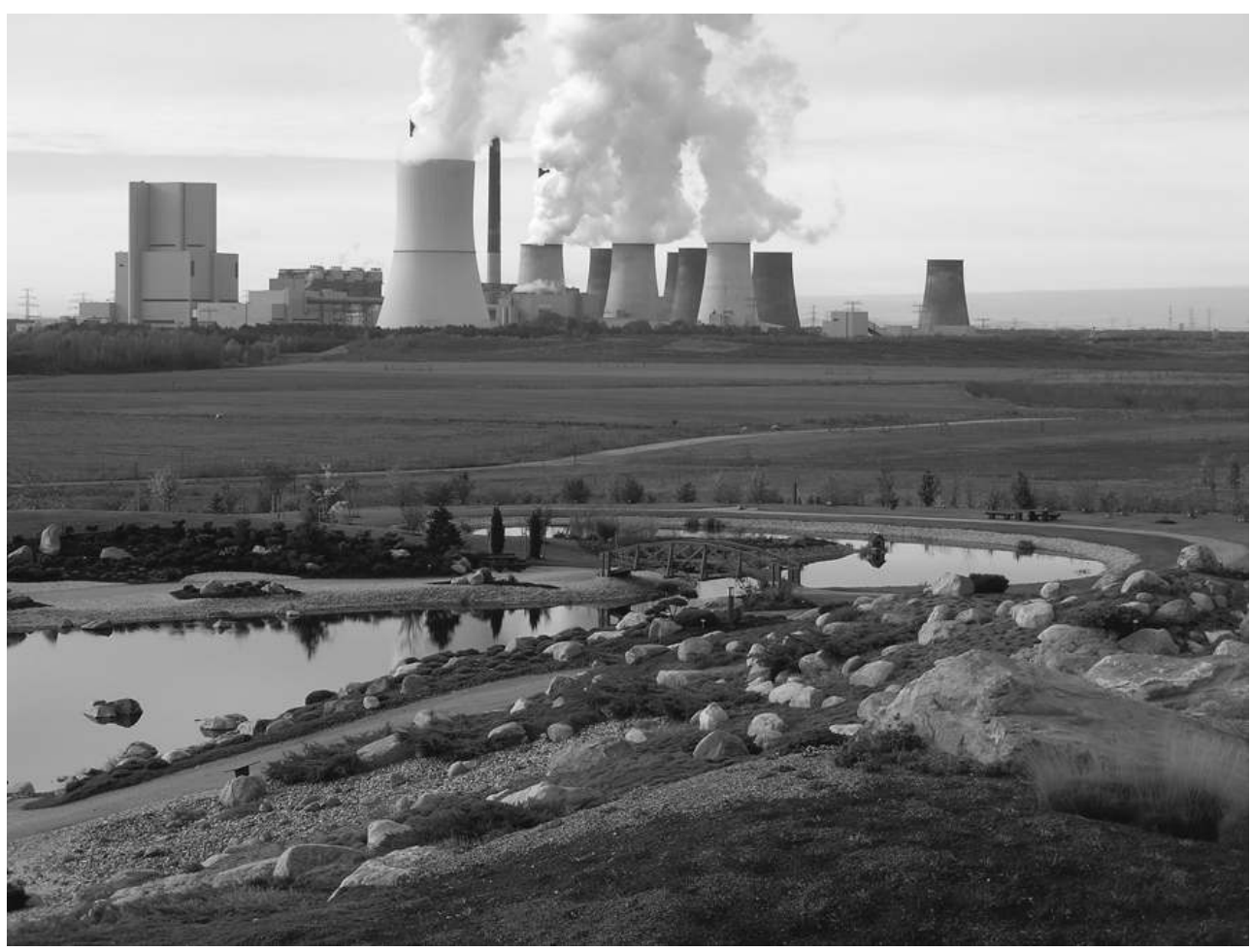

Il ne faut cependant pas exagérer les possibilités de diminuer les rejets de $\mathrm{CO} 2$ par la rénovation du parc de centrales thermiques. Dans son étude déjà citée, la DPG a estimé la réduction possible des émissions de $\mathrm{CO} 2$ si, d'ici à 2020, on remplace la moitié du parc actuel de centrales thermiques; ce qui suppose évidemment des investissements considérables. Dans cette hypothèse, et à condition de conserver le même mixe énergétique qu'actuellement, à production d'électricité constante, les émissions de $\mathrm{CO} 2$ pourraient diminuer de $12 \%$. Mais si l'on se place dans l'hypothèse beaucoup plus probable d'une augmentation de la consommation à un rythme inférieur à celui constaté entre 1993 et 2006 ( $+20 \%$ d'augmentation de la consommation), alors il apparaît que les gains de rendement réalisés par la rénovation du parc de centrales thermiques permettront tout juste de suivre la croissance de la demande sans augmenter les émissions de $\mathrm{CO} 2$.

31 En sortant du nucléaire, l'Allemagne risque donc d'avoir beaucoup de problèmes à atteindre les objectifs qui lui sont assignés dans le cadre du protocole de Kyoto sur la 
réduction des émissions de gaz à effet de serre. En effet, si l'Allemagne a pu, entre 1990 et 2003, réduire ses émissions de $\mathrm{CO} 2$ de $18,5 \%$, c'est essentiellement grâce à l'effondrement industriel de l'ex-RDA et à la diminution drastique de la production de lignite qui en a résulté. Par contre, on peut se demander comment elle pourra continuer à réduire ses émissions de $\mathrm{CO} 2$ d'ici à 2008-2012, alors qu'elle aura déjà commencé à réduire sa production d'électricité d'origine nucléaire dans des proportions que ne pourront pas compenser les énergies renouvelables. On peut d'ailleurs d'ores et déjà constater que depuis 1999 les émissions de CO2 de l'Allemagne ne diminuent plus et ont même augmenté dans le secteur de la production d'électricité, en raison du recours accru au lignite et au gaz naturel (figure 12).

Figure 12 : Évolution des émissions de $\mathrm{CO} 2$ en Allemagne par grand secteur de consommation d'énergie (en millions de tonnes par an) (source : BMWT). Le secteur de l'énergie correspond pour l'essentiel à la production d'électricité qui représentait $41 \%$ des émissions en 1990 et $43 \%$ en 2004.

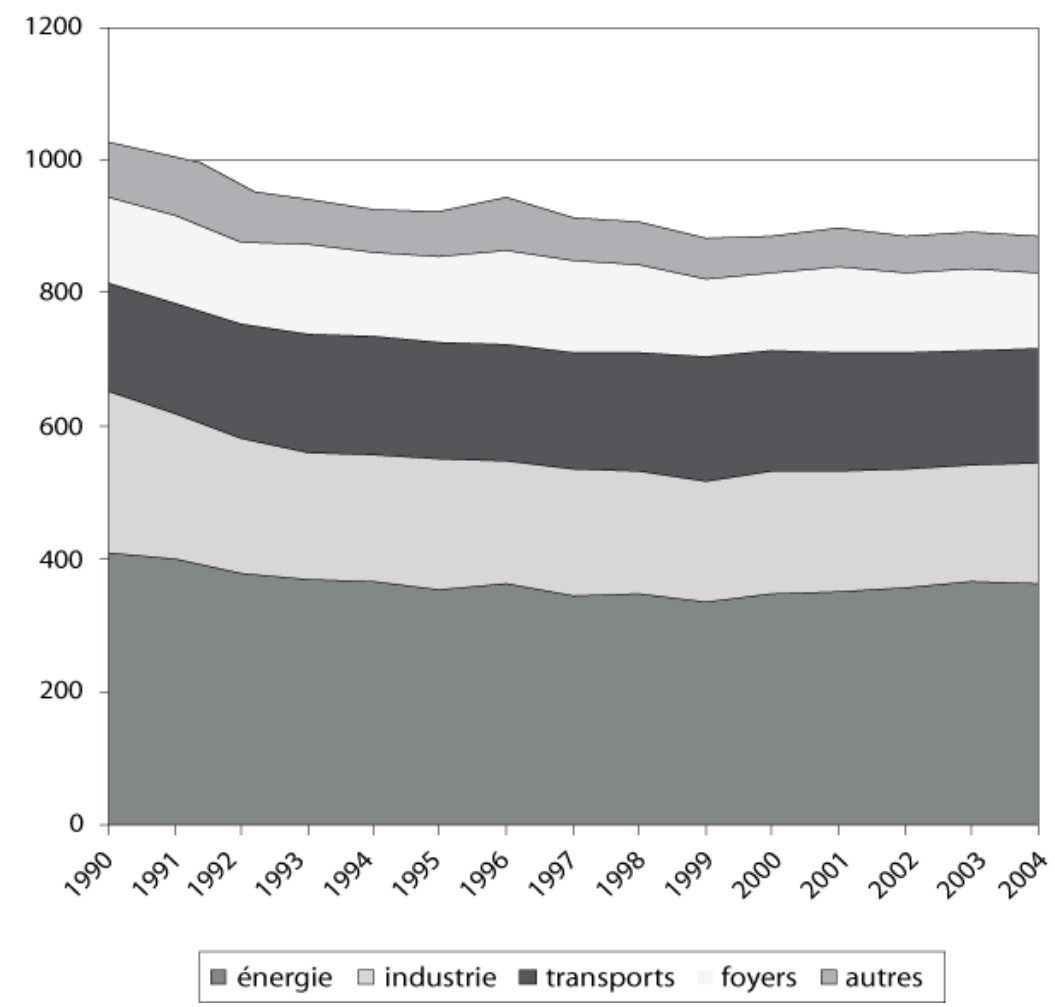

En se plaçant dans l'hypothèse d'un arrêt complet des centrales nucléaires et d'un doublement de la part du gaz naturel et de celle des énergies renouvelables d'ici à 2020, la DPG (2005) a ainsi estimé que, dans le meilleur des cas, cela conduirait à une augmentation des rejets de $\mathrm{CO} 2$, de 54 à 61 millions de tonnes, soit près de $6 \%$ des émissions de 2002. Encore faut-il préciser que cette étude se plaçait dans le cas hautement improbable où la consommation d'électricité resterait constante jusqu'en 2020. C'est pourquoi, en conclusion de son étude, la DPG recommande-t-elle de prolonger le fonctionnement des centrales nucléaires au-delà de 2020, seul moyen pour l'Allemagne d'atteindre ses objectifs de réduction des émissions de dioxyde de carbone8. Les sociétés exploitant les centrales nucléaires qui doivent être prochainement fermées viennent d'ailleurs de déposer des demandes de prolongation d'activité. C'est le cas en particulier de la société RWE, dont le réacteur nucléaire Biblis A, devrait théoriquement être arrêté au cours de l'année 2007. Or, cette demande a 
généré des dissensions à l'intérieur du gouvernement de coalition, puisque le refus du ministre socialiste de l'environnement, Sigmar Gabriel, a été ouvertement critiqué par la chancelière Angela Merkel et par le ministre de l'économie CSU, Michael Glos. Le ministre de l'environnement se montre en effet d'autant plus intransigeant, qu'il soupçonne les sociétés exploitant les réacteurs nucléaires de vouloir gagner du temps en attendant les prochaines élections de 2009 , qui pourraient amener au pouvoir un gouvernement prêt à remettre en question la sortie programmée du nucléaire.

C'est d'ailleurs à cette solution qu'est en train de se résoudre un pays comme la Suède qui, dès 1980, avait annoncé la sortie du nucléaire pour 2010. Or, les réacteurs nucléaires produisent près de la moitié du courant électrique et jusqu'à présent, aucune source d'énergie renouvelable de substitution suffisamment importante n'a pu être découverte. En effet, la seule solution serait d'augmenter encore l'équipement hydroélectrique du pays, mais il se trouve que le gouvernement et le parlement suédois ont renoncé en 1998 à la construction de nouveaux barrages, afin de conserver le caractère naturel d'un certain nombre de fleuves. Aussi, face à cette impasse énergétique, il est maintenant question de prolonger l'exploitation de l'énergie nucléaire bien au-delà de 2010. Un seul réacteur a été stoppé en 1999, près de quatre ans après le terme initialement prévu. Par contre, le deuxième réacteur de la centrale de Bärseback, qui devait être mis hors service en 2001, a été finalement prolongé et il n'y a plus de date prévue pour son arrêt. Ce nouveau tournant dans la politique énergétique de la Suède, qui n'est pas sans causer des remous au sein du gouvernement, s'explique par l'obligation dans lequel se trouve le pays de respecter les engagements du protocole de Kyoto. Or, en l'état actuel des choses, la sortie du nucléaire n'est possible qu'en recourant aux énergies fossiles; ce qui aurait pour effet d'augmenter très sensiblement les émissions de gaz à effet de serre d'un pays qui, pour l'heure, est un de ceux qui en émettent le moins.

\section{L'impact environnemental négligé de l'exploitation du lignite}

Un des paradoxes les plus étonnants du débat énergétique en Allemagne est qu'il se limite presque exclusivement au problème des émissions de gaz à effet de serre et à celui des déchets radioactifs générés par l'activité des centrales nucléaires. Les attaques des opposants au nucléaire se concentrent ainsi sur les conditions du transport et du devenir des combustibles irradiés, organisant des manifestations souvent très dures et très coûteuses. La principale polémique actuelle concerne la question de l'enfouissement des déchets nucléaires ultimes qu'il est prévu de réaliser dans l'ancienne mine de sel de Gorleben, en Basse Saxe. Face aux manifestations écologistes, les gouvernements allemands successifs ont préféré reculer et ont gelé un projet qu'il sera pourtant d'autant plus nécessaire de mener à bien en raison du démantèlement des centrales nucléaires.

La concentration du débat sur la question du nucléaire et des menaces que celui-ci fait peser sur l'environnement fait, qu'assez paradoxalement, les problèmes générés par l'exploitation du lignite sont relégués au second plan, alors que du point de vue environnemental et même social, les nuisances causées par cette exploitation sont infiniment supérieures. Il faut en effet souligner qu'en dehors des émissions de gaz à effet de serre générées par la combustion des énergies fossiles, l'extraction du lignite en Allemagne se fait par le recours à une technique qui provoque des bouleversements 
considérables de l'environnement et des paysages, l'extraction en découverte par des excavatrices géantes (photo 3). Exploité dans des régions densément peuplées, comme la Baie de Cologne en Rhénanie, l'exploitation du lignite nécessite la destruction de villages et même de petites villes dont la population est déplacée au fur et à mesure de la progression des excavatrices. Cette exploitation atteignait certes une ampleur particulière en ex-RDA ou plus de 80000 personnes ont dû ainsi quitter leur village entre 1949 et 1989 et à ce titre elle a été largement dénoncée, notamment à cause de l'effroyable pollution de l'air que générait la combustion du lignite. En conséquence, la production a été considérablement réduite depuis la réunification, si bien que désormais, les problèmes environnementaux que pose cette exploitation dans les nouveaux Länder sont d'ampleur relativement limitée.

Photo 3 : L'exploitation de lignite de Garzweiler, dans la Baie de Cologne est l'une des trois exploitations en activité dans cette région (photo $M$. Deshaies, 2002). Son extension doit dans l'avenir, provoquer le déplacement de 8000 personnes.

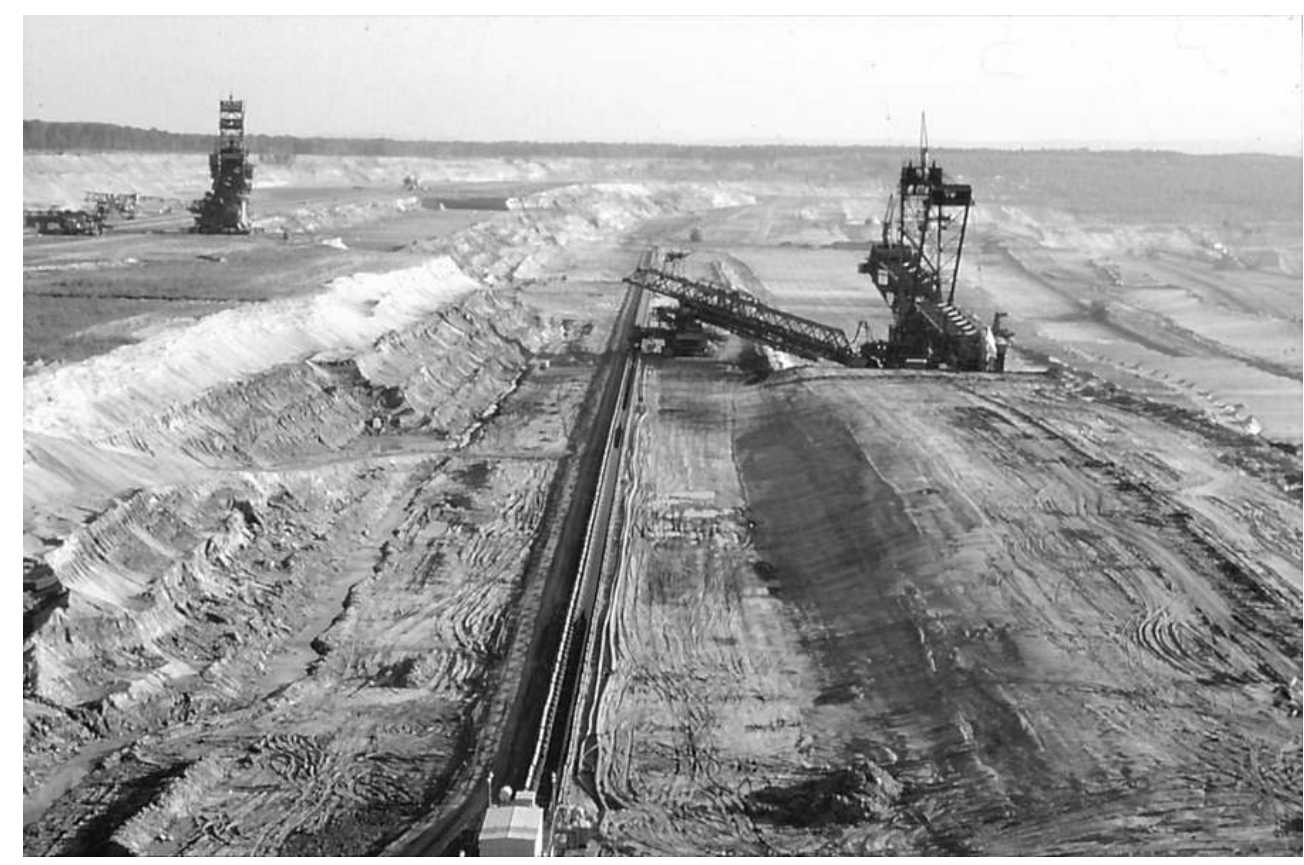

Par contre, les problèmes que pose l'exploitation du lignite dans la région de Cologne sont assez largement occultés dans le débat national concernant l'impact environnemental de la politique énergétique. En effet, sans atteindre l'ampleur de l'exploitation en ex-RDA, l'extraction du lignite dans la région de Cologne a tout de même conduit à déplacer plus de 30000 habitants. Or, contrairement à la situation dans les nouveaux Länder, la réunification n'a eu aucune conséquence sur l'évolution de l'exploitation du lignite qui se maintient à un niveau constant d'environ 100 millions de tonnes par an. Elle se fait surtout à une échelle inégalée dans le passé, à l'image de l'exploitation de Hambach qui dépasse $400 \mathrm{~m}$ de profondeur pour une superficie de plus de 3000 ha (soit environ $5 \mathrm{~km}$ sur 6). Dans l'avenir, l'extension des trois grandes exploitations en activité dans la Baie de Cologne devrait entraîner le déplacement de 15000 personnes et le projet d'extension de l'exploitation de Garzweiler II a généré de vives querelles à l'intérieur de l'ancien gouvernement de Rhénanie du Nord-Westphalie constitué alors par une coalition rouge-verte. Le projet de Garzweiler II continue aujourd'hui à soulever une forte opposition locale de la part des défenseurs de 
l'environnement comme « BUND, Freunde der Erde », et tout simplement des habitants concernés. Cette association de protection de l'environnement a créé un site internet (www.bund-nrw.de/braunkohle) très riche, sur lequel elle diffuse beaucoup d'informations sur les conditions de l'exploitation du lignite, en dénonçant vigoureusement les rejets de gaz à effet de serre de la combustion du lignite et surtout la destruction des paysages que provoque son exploitation. Pourtant, force est de constater que cette opposition locale ne trouve guère de résonance au niveau national puisque, loin de s'engager sur la voie d'une réduction de la production du lignite, l'ancien gouvernement allemand de la coalition rouge-verte a implicitement choisi, en sortant du nucléaire, de confirmer la place du lignite comme un pilier essentiel de la production d'électricité. Les plans d'exploitation en cours prévoient d'ailleurs la poursuite de celle-ci jusqu'à l'horizon 2030-2040.

Il est vrai aussi que les sociétés minières exploitant le lignite peuvent faire valoir qu'il s'agit d'une ressource nationale abondante (au moins 300 ans de réserve), rentable économiquement (c'est-à-dire sans subventions) et qu'après la fin de l'extraction, elles reconstituent des paysages nouveaux de forêts, de cultures et de lacs qui ne manquent pas d'harmonie (photos 4 et 5) et qu'elles présentent dans leurs brochures comme plus "variés » et à la rigueur, plus intéressants et plus attractifs que les paysages initiaux. Les sociétés minières se targuent aussi de leur contribution à la protection de la nature en développant des projets de réhabilitation (Rekultivierung) des paysages, où certaines espèces animales menacées retrouvent une place qu'elles avaient perdue dans les paysages agricoles antérieurs à l'exploitation minière. Pour le cas de la Baie de Cologne en Rhénanie, les projets de réhabilitation paysagère en cours sont présentés sur le site : www.forschungsstellerekultivierung.de. En fait, elles présentent leur action comme une contribution au développement durable; ce qui peut apparaître quelque peu paradoxal. 
Photo 4 : L'exploitation de lignite de Inde, dans la Baie de Cologne (M. Deshaies, 2006). Au premier plan, la rivière Inde qui vient d'être déplacée.

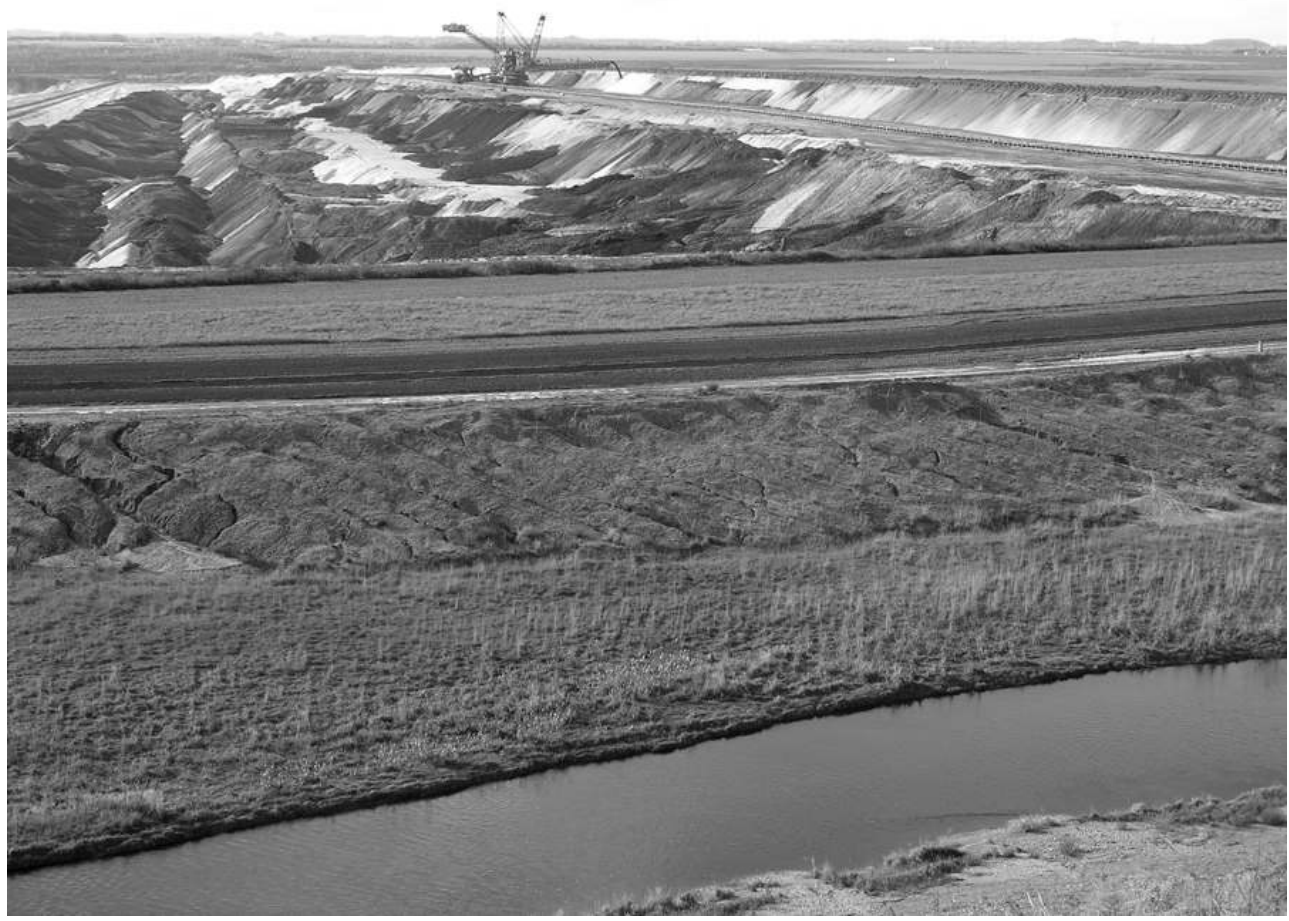

Photo 5 : La rivière Inde déplacée et « renaturée » (M. Deshaies, 2006). La prise de vue est perpendiculaire à la photo 4 .

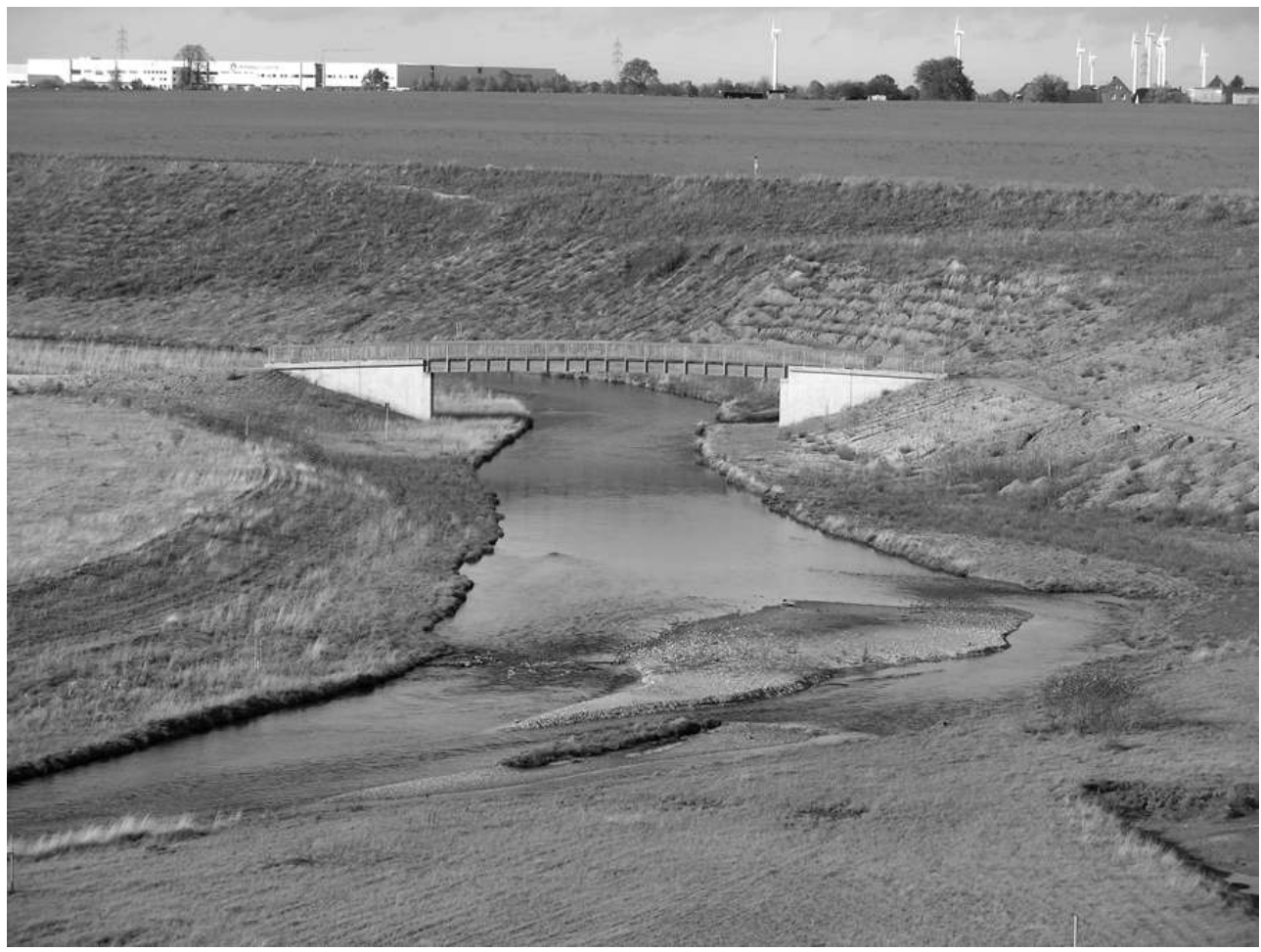

Il faut en effet souligner que, si les plus anciennes reconstitutions paysagères réalisées dans la région de Cologne sont effectivement devenues des aires de loisirs de proximité 
(Naherholungsgebiet) très prisées, les futurs lacs qui occuperont les exploitations minières actuellement en activité seront d'une échelle sans commune mesure avec ceux existant actuellement. Après sa fermeture en 2045, l'exploitation de Hambach, dans la Baie de Cologne, sera transformée en un lac de $300 \mathrm{~m}$ de profondeur pour une superficie de 4200 ha. Le remplissage d'une telle fosse et l'aménagement de ses rives seront évidemment problématiques et poseront un véritable défi aux aménageurs. Enfin, on peut aussi se demander si, quelle que soit la qualité des reconstructions paysagères réalisées par les exploitants miniers, elles constituent vraiment une "indemnité » ou un produit de substitution équitable au "Kulturlandschaft», le paysage ancien façonné, retouché petit à petit par des générations de paysans et qui contient une multitude de repères et de signes culturels irremplaçables.

\section{Conclusion}

39 L'Allemagne est incontestablement un pays où la société attache une grande importance aux questions environnementales. C'est notamment pour cette raison qu'elle a infléchi considérablement sa politique énergétique en s'engageant dans la sortie du nucléaire et en développant fortement les énergies renouvelables et en premier lieu l'énergie éolienne. Dans le même temps, elle s'est engagée avec résolution à atteindre les objectifs particulièrement ambitieux qui lui sont fixés par le protocole de Kyoto, à savoir une diminution de $21 \%$ de ses émissions de gaz à effet de serre entre 1990 et 2008-2012. Mais si elle est d'ores et déjà presque parvenue à remplir ses objectifs, c'est essentiellement grâce à la désindustrialisation de l'Est survenue après la réunification. Le problème est que pour continuer à réduire ses émissions de gaz à effet de serre, elle ne peut plus compter désormais que sur le développement des énergies renouvelables et sur l'amélioration du rendement des centrales thermiques. Or, cela risque bien de se révéler impossible, alors que la production électronucléaire doit diminuer fortement après 2010 et que la consommation d'électricité a augmenté de 20 \% depuis 1993; si bien que même un essor encore plus important des énergies renouvelables ne permettra pas de couvrir les besoins.

Dans ce contexte, les choix énergétiques opérés depuis 1998 conduisent, d'une part à accroître fortement la dépendance énergétique du pays en recourant de plus en plus au gaz naturel et au charbon importés, d'autre part à augmenter les émissions de gaz à effet de serre du fait de l'utilisation accrue du lignite et du charbon. De plus, les nuisances environnementales générées par l'exploitation du lignite apparaissent comme singulièrement absentes du débat national sur la politique énergétique (Deshaies 2004a).

41 Le nouveau gouvernement allemand issu des élections de septembre 2005 et dirigé par Angela Merkel, a bien conscience de la nécessité de revoir la politique énergétique du pays, non seulement pour l'adapter au contexte du renchérissement et à terme de la raréfaction des hydrocarbures, mais aussi dans la perspective du respect des engagements de Kyoto. C'est dans cette optique que la nouvelle chancelière a lancé en avril 2006, un «Energiegipfel » destiné à définir jusqu'en 2020 un concept global de la politique énergétique allemande. Trois groupes de travail ont été créés afin d'examiner les aspects nationaux et internationaux de la politique énergétique, ainsi que la question de l'accroissement de l'efficience énergétique. Ces groupes de travail doivent rendre leurs rapports au printemps 2007 et en fonction de ceux-ci le gouvernement 
allemand définira le concept global de la politique énergétique dans la deuxième moitié de l'année 2007.

Des rapports intermédiaires présentant les premières réflexions de ces groupes de travail ont été rendus publics à la fin du mois de septembre 2006 et sont consultables sur le site du ministère de l'environnement (http://www.bmu.de). Le groupe de travail consacré aux aspects nationaux de la politique énergétique a souligné la nécessité de réévaluer le thème de la sûreté des approvisionnements énergétiques. Il semble unanime pour préconiser le maintien du rôle du charbon et du lignite dans la production d'électricité et estime possible un doublement de la productivité énergétique en 2020 par rapport à 1990. Par contre, des divergences sont soulignées en ce qui concerne la place future du nucléaire et des énergies renouvelables. Si une partie du groupe de travail estime possible une sortie du nucléaire, sans que cela entraîne un accroissement des rejets de dioxyde de carbone et des prix du courant électrique, des voix dissonantes jugent qu'il est impossible de renoncer au nucléaire, notamment parce qu'il n'est pas économiquement envisageable que les énergies renouvelables puissent représenter $20 \%$ de la production de courant électrique.

Il y a donc tout lieu de penser que la contradiction insurmontable entre la sortie du nucléaire et les objectifs de diminution des émissions de gaz à effet de serre est susceptible d'amener à un nouveau tournant dans la politique énergétique du pays, comme cela s'est produit récemment dans d'autres pays européens.

\section{BIBLIOGRAPHIE}

BGR (2006). - Bundesanstalt für Geowissenschaften und Ressourcen. - Reserven, Ressourcen und Verfügbarkeit von Energierohstoffen 2006, Hannover.

BRÜCHER W. (2006). - Naturgegebene und anthropogene Grenzen der erneuerbaren Energien, Der Erdkundelehrer, Nr 25, S. 9-17.

BRÜCHER W. \& HELFER M. (2004). - Energienachfrage und Angebotsdifferenzierung, in :

Nationalatlas Bundesrepublik Deutschland, Band 8, Unternehmen und Märkte, Leibnitz Institut für Länderkunde, p. 130-133.

DEBRIV (2003). - Bundesverband Braunkohle : Braunkohle 2003 - Ein Industriezweig stellt sich vor, Köln.

DESHAIES M. (2004a). - Energie et paysages en Allemagne : les enjeux environnementaux, Annales de Géographie, n 637, p. 27-51.

DESHAIES M. (2004b). - La question énergétique en Allemagne et en Europe centrale, Revue d'Allemagne, $\mathrm{n}^{\circ} 3 / 4$, p. 323-340.

DESHAIES M. (2006). - Energie et environnement en Europe : la question de la production

d'électricité, L'information géographique, vol. 70, septembre, p. 50-71.

DLR/IFEU/WI (2004). - Ökologisch optimierter Ausbau der Nutzung erneuerbarer Energien in Deutschland 
DPG (2005). - Klimaschutz und Energieversorgung in Deutschland (1990-2020, 101 p.

Energiereport IV (2005). - Die Entwicklung der Energiemärkte bis zum Jahr 2030, EWI/PROGNOS, Kurzfassung, $43 \mathrm{p}$.

EWI/PROGNOS (2006). - Auswirkungen höherer Ölpreise auf Energieangebot und -nachfrage, Langfassung, $181 \mathrm{p}$.

Gesamtverband des deutschen Steinkohlenbergbaus (2005). - Die Zukunft braucht Kohle, Steinkohle Jahresbericht, $80 \mathrm{p}$.

Gesamtverband des deutschen Steinkohlenbergbaus (2006). - Sicherheit für Morgen, Steinkohle Jahresbericht, $71 \mathrm{p}$.

GÖTZ R. (2006). - Deutschland und Russland - «strategische Partner» ?APuZ, p. 14-23.

HEURIAUX C. (2002). - Le marché énergétique allemand, éditions du Temps, 252 p.

ISET (2004). - Windenergiereport Deutschland, Kassel, DENA-Studie.

LÜBKE R. \& VAN DE LOO K. (2007). - Energiepolitische Perspektiven für Europa und Deutschland und die Rolle der Steinkohle im Energiemix, Glückauf, GVST, S. 69-74.

Nordrheinwestfälische Akademie der Wissenschaften (2006). - Die Energieversorgung sichern, Denkschrift, $38 \mathrm{~S}$.

Publications en ligne

BMU (2005). - Bericht der Bundesrepublik Deutschland 2005 zur Erreichung des Richtziels für den Verbrauch von Strom aus Erneuerbaren Energiequellen im Jahr 2010, http://www.bmu.de BMU (2006a). - Energieversorgung für Deutschland, Statusbericht für den Energiegipfel am 3. April 2006, http://www.bmu.de

BMU (2006b). - Umweltbewusstsein in Deutschland 2006, 80 p., http://www.umweltdaten.de/ publikationen/fpdf-l/3113. pdf

BMWT, Bundesministerium für Wirtschaft und Technologie (2006). - Energieversorgung für Deutschland, 74 p. (http://www.bmwi.de/BMWI/Redaktion/PDF/E/energiegipfel-statusbericht, property=pdf, bereich=bmwi, sprache=de, $r w b=$ true.pdf)

DESHAIES M. (2003). - Energie et environnement en France et en Allemagne, www.deuframat.de

\section{Sites internet consultés}

Bundesanstalt für Geowissenschaften und Rohstoffe : http://www.bgr-bund.de, consulté le 19 mars 2007

BUND, Freunde der Erde : http://www.bund-nrw.de/braunkohle.htm

http://www.zukunft-statt-braunkohle.de, consultés le 17 mars 2007

Bundesministerium für Wirtschaft und Technologie : http://www.bmwi.de, consulté le 25 mars 2007

Bundesverband Braunkohle : http://www.debriv.de, consulté le 17 mars 2007

Bundesumweltministerium : http://www.bmu.de, consulté le 18 mars 2007

Bundesverband Windenergie : http://www.wind-energie.de/

de/statistiken/, consulté le 17 mars 2007 
Butendiek Offshore Windpark : http://www.butendiek.de, consulté le 18 mars 2007

DEWI, Deutsches Windenergie-Institut : http://www.dewi.de, consulté le 17 mars 2007

Eurostat : http://epp.eurostat.ec.europa.eu, consulté le 18 mars 2007

Gesamtverband des deutschen Steinkohlenbergbaus : http://www.gvst.de, consulté le 17 mars 2007

Forschungsstellerekultivierung : http://forschungsstellerekultivierung.de, consulté le 18 mars 2007

Stiftung Offshore Windenergie : http://www.offshore-stiftung.de, consulté le 18 mars 2007

Umweltbundesamt : http://wwwumweltdaten.de/publikationen/fpdf-1/3113.pdf, consulté le 17 mars 2007

\section{RÉSUMÉS}

À partir des années 1980, l'importance croissante des questions environnementales a infléchi considérablement la politique énergétique allemande, conduisant à une remise en question de l'utilisation du nucléaire. La réduction de la production d'électricité résultant de la décision de sortie du nucléaire doit être compensée par une diminution de la consommation et par le développement des énergies renouvelables et en particulier de l'électricité produite à partir des éoliennes. Mais les limites au développement des énergies renouvelables, ainsi que l'évolution de la consommation rendent de moins en moins probable la réalisation d'un tel scénario. La sortie du nucléaire devrait conduire à un accroissement sensible de la consommation d'énergies fossiles, en particulier le gaz naturel, le lignite et le charbon. Outre l'accroissement de la dépendance énergétique du pays, cette évolution risque de provoquer une augmentation des émissions de gaz à effet de serre, ce qui est en contradiction avec les engagements environnementaux de l'Allemagne dans le cadre du Protocole de Kyoto et devrait conduire à un nouveau tournant énergétique.

Since the 80ies the importance of environmental isssues and concerns have bent the German energy policy which have left this energy solution. The decrease of the electric production resulting from the coming out of the nuclear solution must be counter-balanced with the growth of renewable energies, particularly windmill production. The limits of these renewable energies and the growth of the consumption. As a consequence, the out of the nuclear solution will strenghten consumption of fossil energies, particularly gas, lignite and coal. The risks are not only an increase of energy dependence, but also huge glashouse affect gas emissions as a result. This evolution is inconsistent with the environmental obligations of Germany within Kyoto Protocol and could lead again to an energy turning.

Seit den 80er Jahren hat die steigende Bedeutung der umweltrelevanten Probleme in Deutschland dessen Energiepolitik beträchtlich beeinflusst, was dazu führte, dass die Nutzung der Atomenergie in Frage gestellt wurde. Die aufgrund des beschlossenen Atomausstiegs sinkende Produktion von Elektrizität muss folglich kompensiert werden sowohl durch Einsparungen als auch durch entsprechende Entwicklung erneuerbarer Energiequellen, besonders der Windenergie. Doch lassen die begrenzten Möglichkeiten der Erneuerbaren sowie die voraussichtlich steigende Verbrauchskurve ein solches Szenario als immer weniger realistisch erscheinen. Vielmehr würde der Atomausstieg zu einer merklich anwachsenden Nutzung der fossilen Energieträger führen, vor allem von Erdgas, Braunkohle und Steinkohle. Abgesehen von der dadurch gesteigerten Energieabhängigkeit Deutschlands, würde es mit einer 
solchen Entwicklung riskieren, mehr Treibhausgase zu emittieren und sich damit in Widerspruch zu seinen umweltpolitischen Verpflichtungen im Rahmen des Kyoto-Protokolls begeben, ja sogar eine energiepolitische Kehrtwende einleiten.

INDEX

Keywords : out of the nuclear solution, renewable energy, fossil energy, energy dependence, glashouse affect gas emissions

Schlüsselwörter : Atomausstieg, erneuerbare Energien, fossile Energieträger, Energieabhängigkeit, Treibhausgase

Mots-clés : sortie du nucléaire, énergie renouvelable, énergie fossile, dépendance énergétique, gaz à effet de serre

\section{AUTEUR}

\section{MICHEL DESHAIES}

Centre d'Etudes et de Recherche sur les Paysages - Université de Nancy 2 - 23, bd Albert Ier, 54015 Nancy CEDEX 